\title{
Spatiotemporal dynamics of EEG microstates in four- to eight- year-old children: Age- and sex-related effects
}

Armen Bagdasarov ${ }^{\mathrm{a}}$, Kenneth Roberts ${ }^{\mathrm{b}}$, Lucie Bréchet ${ }^{\mathrm{c}}$, Denis Brunet ${ }^{\mathrm{c}, \mathrm{d}}$, Christoph M. Michel ${ }^{\mathrm{c}, \mathrm{d}}$, Michael S. Gaffrey ${ }^{\mathrm{a}}$

aDepartment of Psychology \& Neuroscience, Duke University, Reuben-Cooke Building, 417 Chapel Drive, Durham, NC, 27708, USA

${ }^{b}$ Duke Institute for Brain Sciences, Duke University, 308 Research Drive, Durham, NC, USA

'Department of Basic Neurosciences, University of Geneva, Campus Biotech, 9 Chemin des Mines, 1202 Geneva, Switzerland

${ }^{\mathrm{d} C e n t e r}$ for Biomedical Imaging (CIBM) Lausanne, EPFL AVP CP CIBM Station 6, 1015 Lausanne Switzerland

* Corresponding author email: armen.bagdasarov@duke.edu

Co-author emails: ken.roberts@duke.edu, lucie.brechet@unige.ch, denis.brunet@unige.ch, christoph.michel@unige.ch,michael.gaffrey@duke.edu

CRediT - Authorship Contribution Statement: Bagdasarov: Conceptualization, Methodology, Software, Validation, Formal Analysis, Data Curation, Writing - Original Draft, Writing - Review \& Editing, Visualization. Roberts: Software, Validation. Bréchet: Software, Validation, Writing - Review \& Editing. Brunet: Software, Validation. Michel: Software, Validation, Writing Review \& Editing. Gaffrey: Conceptualization, Methodology, Resources, Writing - Review \& Editing, Supervision, Funding Acquisition.

Declaration of competing interests: The authors declare that they have no known competing financial interests or personal relationships that could have appeared to influence the work reported in this paper.

Data statement: The data that support the findings of this study are available on request from the corresponding author. The data are not publicly available due to privacy or ethical restrictions.

Acknowledgements: This study was funded by a grant from the National Institute of Mental Health (R01MH110488) awarded to Michael S. Gaffrey. 
Keywords: EEG Microstates, Resting-State Networks, Brain Development, Children, Age, Sex Differences

\begin{abstract}
The ultrafast spatiotemporal dynamics of large-scale neural networks can be examined using resting-state EEG microstates, representing transient periods of synchronized neural activity that evolve dynamically over time. In adults, four canonical microstates have been shown to explain most topographic variance in resting-state electroencephalography (EEG). Their temporal structures are age-, sex- and state-dependent, and are susceptible to pathological brain states. However, no studies have assessed the spatial and temporal properties of EEG microstates exclusively during early childhood, a critical period of rapid brain development. Here we sought to investigate EEG microstates recorded with high-density EEG in a large sample of 103, 4-8-yearold children. Using data-driven $k$-means cluster analysis, we show that the four canonical microstates reported in adult populations already exist in early childhood. Using multiple linear regressions, we demonstrate that the temporal dynamics of two microstates are associated with age and sex. Source localization suggests that attention- and cognitive control-related networks govern the topographies of the age- and sex-dependent microstates. These novel findings provide unique insights into functional brain development in children captured with EEG microstates.
\end{abstract}




\section{Introduction}

Early childhood is a period of rapid brain development and behavioral change. Characterizing the developmental properties of network-based brain connectivity during early childhood is likely to critically inform our understanding of normative and atypical patterns of neurocognitive development. One electroencephalography (EEG) method that has emerged as highly useful for characterizing the spatiotemporal dynamics of large-scale brain networks is microstate analysis (for reviews, see Khanna et al., 2015 and Michel \& Koenig, 2018). EEG microstates (Lehmann et al., 1987) are patterns of scalp potential topographies that transition between each other every 60-120 milliseconds (ms) and reflect transient periods of synchronized neural activity that evolve dynamically over time (Michel \& Koenig, 2018). Importantly, recent research suggests that reliable estimates of microstates and their properties can be derived from just two minutes of EEG data collection (Liu et al., 2020); a critical advance when working with sensitive and young research groups. Nevertheless, few studies to date have used this approach in children, and none have focused exclusively on this early developmental period. As a result, the potential to provide unique insights into brain development during the earliest years of life by combining the practical nature of EEG data collection with the analytical approach of microstate analysis remains largely unexplored.

Previous studies in older age groups using clustering methods have reported that four microstates - canonically labeled A, B, C, and D - explain the majority of variance in scalp potential topography of resting-state EEG (Michel \& Koenig, 2018). Simultaneous EEGfunctional magnetic resonance imaging (fMRI) studies as well as EEG source imaging have shown that the spatial patterns of these microstates resemble well-known resting-state networks (RSNs; 
e.g., microstate A representing auditory, B representing visual, $\mathrm{C}$ representing salience, and D representing attention networks) (Bréchet et al., 2019; Britz et al., 2010; Custo et al., 2017). Existing research indicates that rapid transitions between microstates reflect the dynamic reorganization of large-scale functional networks (Michel \& Koenig, 2018; Ville et al., 2010). Importantly, the millisecond temporal resolution of EEG allows unique information about the temporal properties of each microstate to be quantified, including its global explained variance (GEV; i.e., percentage of total variance in the data explained by a given microstate), average duration, percentage of time for which it is present (i.e., coverage), and frequency of occurrence per second. As a result, prior studies support EEG microstate analysis as a novel method for measuring the spatiotemporal properties of network-based connectivity (Lehmann, 2010).

To date, only two studies have examined microstates in children (Koenig et al., 2002; Tomescu et al., 2018), neither of which included typically developing children under the age of six. In a cross-sectional study including participants 6-80 years old, Koenig et al. (2002) found no differences in the temporal parameters between the four canonical microstates in 6-12-year-olds, but comparatively shorter microstate A, B, and D and longer C durations in 12-16-year-olds. Microstates $\mathrm{C}$ and D occurred more frequently and had longer durations than microstates A and B in 12-16-year-olds (Koenig et al., 2002). In 16-21-year-olds, microstate $\mathrm{C}$ was the longest and occurred most frequently - an observation that remained in 21-80-year-olds - while D dropped in duration and occurrence (Koenig et al., 2002). Microstates A and B remained the shortest and least frequent in 21-80-year-olds but occurred more frequently than in 16-21-year-olds (Koenig et al., 2002). Tomescu et sample of 6-87-year-olds. Males compared to females had shorter durations of microstate C. The 
duration of this microstate decreased from the 14-19-year-old period to the 20-30-year-old period in males only, during which males had shorter microstate $\mathrm{C}$ durations than females (Tomescu et al., 2018). Males compared to females had a higher occurrence of Microstate D, and this pattern was present in all age groups except during the 14-19-year-old period (Tomescu et al., 2018). However, Microstate D increased in frequency from the 14-19-year-old period to the 20-30-yearold period for both sexes (Tomescu et al., 2018). Together, these studies suggest age- and sexrelated effects in the spatiotemporal dynamics of resting-state EEG across the lifespan, particularly in the duration and occurrence of microstates $\mathrm{C}$ and $\mathrm{D}$. While supporting the developmental nature of microstates, neither study utilized source localization to determine the brain regions underlying each microstate. As a result, the significance of their findings on functional brain network organization and potential links with behavior in children remain unclear. Furthermore, both studies identified group-level microstates using all participants regardless of age. Thus, some important developmental variations in EEG microstate topographies and temporal dynamics that are specific to children may have been overlooked. Since the brain undergoes developmental changes in its functional organization, especially during early childhood (Brown \& Jernigan, 2012; M. H. Johnson, 2011; Long et al., 2017), these variations may be particularly important.

The current study examined the spatiotemporal characteristics of EEG microstates and age and sex effects in a large group of 103, 4-8-year-old children using high-density EEG collected during eyes-closed rest. Given our use of a data-driven method for identifying microstates (Bréchet et al., 2019; Custo et al., 2017) and the absence of microstate research in young children, we did not make group-level predictions about the number of microstates that would be present, their topographies, or whether the temporal parameters would differ between microstates. However, we 
expected that the temporal parameters would change with increasing age and show sex differences (Tomescu et al., 2018), although we did not make specific hypotheses regarding directionality of effects. We also localized the sources of the microstates and expected them to resemble wellestablished RSNs captured with fMRI or EEG given previous findings in older groups (Bréchet et al., 2019; Britz et al., 2010; Custo et al., 2017). Since the development of cognitive functions is prolonged in children, we expected larger age and sex effects for microstates resembling networks involved in higher-order cognitive functions and network integration compared to microstates resembling motor and sensory networks (Bie et al., 2012). 


\section{Methods}

\subsection{Participants}

Participants were children 4-8 years of age recruited from a database maintained by the Department of Psychology and Neuroscience at Duke University and community events. Recruitment details, including inclusion/exclusion criteria, are provided in the Supplementary Materials. After screening 323 children, 249 were eligible. Of these, 171 children completed their first study visit. Resting-state EEG was added to the study approximately halfway through data collection. The number of eligible children who participated at a time when EEG was part of the study protocol was 140. Thirty-two children did not complete EEG because they were noncompliant (e.g., refused to wear the EEG net). In sum, 108 participants completed an EEG session at either their first or second study visit and 103 of them provided adequate data for analyses (see below). Data collection for the final sample of 103 participants occurred between April 2019 and June 2021. All research was approved by Duke University's Institutional Review Board and carried out in accordance with the Declaration of Helsinki. Caregivers provided informed consent and children provided verbal assent. Compensation was provided for study participation. Participant demographics are described in Table 1. 


\section{Table 1}

Participant demographics.

\begin{tabular}{lll}
\hline & Mean (SD) & Range \\
\hline Age (years) & $6.37(1.07)$ & $4.51-8.65$ \\
Income-to-Needs Ratio & $3.04(1.03)$ & $0.15-4.69$ \\
\hline & $n$ & Percent $(\%)$ \\
\hline Biological Sex & 58 & $56.31 \%$ \\
$\quad$ Females & 45 & $43.69 \%$ \\
$\quad$ Males & & \\
Race & 77 & $74.76 \%$ \\
White & 15 & $14.56 \%$ \\
Mixed & 7 & $6.80 \%$ \\
Black or African American & 2 & $1.94 \%$ \\
Asian & 2 & $1.94 \%$ \\
Other & 0 & $0 \%$ \\
Native Hawaiian or Other Pacific Islander & & \\
Ethnicity & 91 & $88.35 \%$ \\
Not Hispanic or Latino & 12 & $11.65 \%$ \\
Hispanic or Latino & & \\
Maternal Education & 1 & $.97 \%$ \\
High School Diploma or GED & 15 & $14.56 \%$ \\
Some College & 10 & $9.71 \%$ \\
Graduated 2-Year College & 33 & $32.04 \%$ \\
Graduated 4-Year College & 4 & $3.88 \%$ \\
Part Graduate or Professional School & 40 & $38.83 \%$ \\
Graduated from Graduate or Professional School & & \\
\hline
\end{tabular}

Note. The Income-to-Needs Ratio (ITN) was calculated by dividing total family income by a poverty threshold determined by the United States Census Bureau, which considered the year assessed and household family size. 


\subsection{EEG data acquisition and preprocessing}

EEG was recorded using a 128-channel HydroCel Geodesic Sensor Net (Electrical Geodesics, Eugene, OR) at $1000 \mathrm{Hertz}(\mathrm{Hz})$, referenced online to the vertex. Impedances were maintained below 50 kilohms throughout the paradigm, which consisted of eight one-minute blocks of alternating eyes-open and eyes-closed resting-state (i.e., four minutes of each condition). Verbal cues given by the experimenter and text displayed through E-Prime software (Psychological Software Tools, Pittsburgh, PA) instructed participants to relax with their eyes open or closed. Following previous research (e.g., Tomescu et al., 2018), only the eyes-closed condition was analyzed for the current study.

Offline preprocessing was performed with MATLAB (The MathWorks Inc, Natick, MA) and EEGLAB (Delorme \& Makeig, 2004) using custom scripts available on https://github.com/DEEDLabEEG. Detailed steps are provided in the Supplementary Materials. Briefly, channels located on the outer ring of the EEG net were removed. Data were downsampled to $250 \mathrm{~Hz}$, low-pass filtered at $40 \mathrm{~Hz}$, and high-pass filtered at $1 \mathrm{~Hz}$. Remaining electrical line noise at $60 \mathrm{~Hz}$ was attenuated using the CleanLine plugin (Mullen, 2012). Bad channels were removed if they 1) were flat for more than five seconds, 2) contained more than four standard deviations of line noise relative to their signal, or 3) correlated at less than .8 to an estimate based on nearby channels. Artifact Subspace Reconstruction (ASR) removed portions of data containing artifacts (Mullen et al., 2015). Additional data periods were removed if more than $25 \%$ of channels' power exceeded seven standard deviations. Independent component analysis (ICA; Lee et al., 1999) with principal component analysis (30 components) was performed, and the ICA matrix was copied over to the full-length data (i.e., before ASR removed artifacted portions). The ICLabel 
plugin (Pion-Tonachini et al., 2019) removed components with a probability greater than .7 of being eye or muscle artifacts. Data were segmented into nonoverlapping one-second epochs and removed using the TBT plugin (Ben-Shachar, 2018) if: At least 10 channels with 1) amplitudes greater than 100 microvolts $(\mu \mathrm{V})$ or less than $-100 \mu \mathrm{V}$, or 2) joint probabilities above three standard deviations for local/global thresholds. If less than 10 channels met rejection criteria, the epoch was not removed, but the channels were interpolated for that epoch only. Lastly, channels removed previously were interpolated using spherical splines, and all channels were re-referenced to the average.

After preprocessing and removing five participants whose data did not pass quality control (see Supplementary Materials, Supplementary Table 1), the minimum amount of data across 103 participants was 145 seconds. To reduce potential effects of varying data lengths across participants on further analyses, data for all participants were trimmed to their first 145 seconds, exceeding the previously published two-minute mark for the reliability of microstate analysis (Liu et al., 2020).

\subsection{Microstate analysis}

Microstate analysis was performed with Cartool (Brunet et al., 2011), first at the individuallevel and then at the group-level. At the individual-level, a spatial filter (Michel \& Brunet, 2019) was applied to each participant's EEG data to remove topographic outliers and smooth topographies. Fifty smaller resampled epochs covering $99.9 \%$ of each participant's data were created. For each epoch, topographies at global field power (GFP) peaks representing timepoints of the highest signal-to-noise ratio (Brunet et al., 2011) were submitted to a polarity-invariant 
modified $k$-means cluster analysis (Pascual-Marqui et al., 1995), which was set to repeat 50 times and identify 1-12 clusters of topographies for each participant.

A second $k$-means cluster analysis was performed at the group-level. This time, 100 smaller resampled epochs, covering $99.7 \%$ of the topographies identified at the individual-level were submitted to a polarity-invariant modified $k$-means cluster analysis, which was set to repeat 100 times and identify 1-15 clusters of topographies. The optimal number of clusters of topographies - now referred to as the group-level microstates - was determined by an aggregate measure of seven independent criteria (Bréchet et al., 2019; Custo et al., 2017).

These microstates were backfitted to each participant's original spatially filtered data, including all data points (not just at GFP peaks). The data was normalized by the median of GFP to account for individual differences in scalp potential due to varying skull conductivity. Backfitting involved calculating the spatial correlation between each microstate at the group-level and each individual data point for each participant, such that the microstate with the highest correlation was assigned to that data point. The polarity of maps was ignored when calculating the correlation. The minimum correlation for data points assigned to a microstate was 50\%. After backfitting, temporal smoothing (window half-size of $32 \mathrm{~ms}$, Besag factor of 10; Pascual-Marqui et al., 1995) was applied, and the removal of improbably small segments. The backfitting procedure produced values of each microstate's GEV, duration, coverage, and occurrence.

\subsubsection{Source localization of microstates}


Six thousand solution points were distributed equally in a grey matter-constrained head model of a child MRI brain volume template. The EEG net template was co-registered to the MRI head model. The Local Spherical Model with Anatomical Constraints (LSMAC; Brunet et al., 2011) calculated an adaptive local spherical model at each electrode by estimating the thicknesses of the scalp, skull, cerebrospinal fluid, and brain under each electrode. These thicknesses were then used in a 4-shell spherical model with local radiuses, allowing the real geometry between solution points and electrodes to be accounted for. A distributed linear inverse solution, LORETA (Low Resolution Brain Electromagnetic Tomography; Pascual-Marqui et al., 1994), was calculated. The results were optimized with regularization, which accounted for background EEG noise and enforced smoothness of the results, and were standardized to correct for the variability of EEG power across time (Michel \& Brunet, 2019). The amplitude of dipoles was saved as scalar, positive values at each solution point and averaged across timepoints for each microstate.

Each microstate's source map was thresholded to the solution points above the 95th percentile of activations across participants (Bréchet et al., 2020, 2021). Source maps were converted to volumes and imported to the Analysis of Functional NeuroImages (AFNI; Cox, 1996) program. In AFNI, they were demeaned by subtracting the mean of all source maps from each source map to highlight microstate-specific sources (Custo et al., 2017). Additional information is provided in the Supplementary Materials.

\subsection{Statistical analyses}

Statistical analyses were conducted in $R$ (R Core Team, 2021) and conceptualized as exploratory. Four one-way, repeated-measures, equal- $n$, Type II sum of squares, analysis of 
variance (ANOVA) models were computed to compare the mean of each temporal parameter (GEV, duration, coverage, occurrence) between microstates. Extreme outliers were determined using boxplots; values above Q3+3*IQR or below Q1-3*IQR (IQR = Interquartile Range) were identified and removed for each ANOVA. If the assumption of sphericity was violated according to Mauchly's test, the Greenhouse-Geisser correction was applied. For each statistically significant ANOVA, post-hoc paired $t$-tests were performed between microstates, and $p$ values were Benjamini-Hochberg-corrected for six comparisons (Benjamini \& Hochberg, 1995). We also calculated estimation statistics-based effect sizes and confidence intervals (CIs) using the DABEST package (Bernard, 2019; Claridge-Chang \& Assam, 2016; Ho et al., 2019). Effect sizes were calculated as the paired mean difference between comparisons. Bias-corrected, accelerated 95\% CIs of the paired mean differences were calculated by performing nonparametric bootstrap resampling (5000 resamples).

Next, separate multiple linear regressions with each microstate's temporal parameter as the dependent variable and mean-centered age, dummy coded sex (males $=-1$, females $=1$, and age by sex interaction as the independent variables were computed (Kraemer \& Blasey, 2004). Multivariate outliers were determined by the Minimum Covariance Determinant for each regression separately and removed (Rousseeuw \& Driessen, 1999). Since microstates are independent of each other and four temporal parameters were assessed for each microstate, $p$ values for the omnibus $F$ test of the overall significance of the complete set of predictors were Benjamini-Hochberg-corrected for four comparisons. Statistically significant interactions were explored by testing whether the slope of age predicting the microstate's temporal parameter was significant for each sex, and the Johnson-Neyman procedure was employed to determine at which 
ages the relationship between sex and the microstate's temporal parameter was significantly different from zero (Johnson \& Neyman, 1936). 


\section{Results}

The meta-criterion for determining the optimal number of microstates revealed four microstates labeled 1, 2, 3, and 4 (Figure 1). In previous literature, microstates 1, 2, and 3 correspond to the canonical A, B, and C, respectively (Michel \& Koenig, 2018). The topography of microstate 4 is less consistent in the previous literature; some studies show a fronto-central maximum while others show a posterior-central maximum (Michel \& Koenig, 2018). Threedimensional views of each microstate and spatial correlations between their topographies are presented in Supplementary Figures 1 and 2, respectively. A mean of 3.40 seconds of data $(\mathrm{SD}=$ 1.76 seconds) across participants did not meet the minimum spatial correlation threshold of $50 \%$ during backfitting to be assigned a microstate. Descriptive statistics of the temporal parameters of the microstates are provided in Table 2. 


\section{Figure 1}

The four microstates.

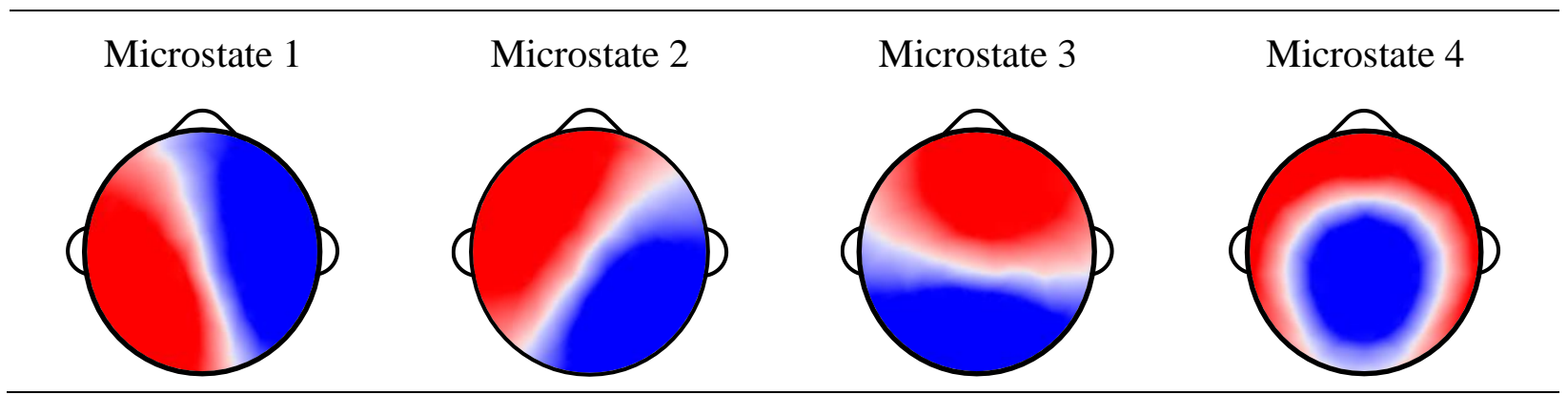

Note. Microstates were derived from a polarity-invariant clustering algorithm.

Table 2

Descriptive statistics of the temporal parameters of the four microstates.

\begin{tabular}{lllll}
\hline & Microstate 1 & Microstate 2 & Microstate 3 & Microstate 4 \\
\hline GEV & & & & \\
$\quad$ Mean (SD) & $.09(.03)$ & $.16(.05)$ & $.28(.08)$ & $.06(.03)$ \\
$\quad$ Range & $.02-.16$ & $.06-.36$ & $.11-.50$ & $.004-.16$ \\
\hline Duration (milliseconds) & & & & \\
$\quad$ Mean (SD) & $78.82(4.59)$ & $85.52(6.97)$ & $100.52(10.95)$ & $74.16(5.91)$ \\
$\quad$ Range & $67.82-94.81$ & $74.23-111.86$ & $77.83-136.40$ & $58.22-87.46$ \\
\hline Coverage (percent) & & & & \\
$\quad$ Mean (SD) & $19.78(4.13)$ & $27.00(5.94)$ & $38.97(7.95)$ & $14.26(5.49)$ \\
$\quad$ Range & $9.63-30.72$ & $13.63-48.86$ & $19.20-58.41$ & $2.15-29.19$ \\
\hline Occurrence (per second) & & & & \\
$\quad$ Mean (SD) & $2.18(0.33)$ & $2.68(0.36)$ & $3.16(0.31)$ & $1.68(0.53)$ \\
$\quad$ Range & $1.14-2.82$ & $1.63-3.45$ & $2.14-3.71$ & $0.35-2.99$ \\
\hline
\end{tabular}




\subsection{Between-microstate differences in the means of their temporal parameters}

Differences in the means of GEV, duration, coverage, and occurrence between the four microstates were examined using one-way repeated-measures ANOVAs with post-hoc contrasts Benjamini-Hochberg-corrected for six comparisons. The results of all models with outliers excluded are presented in Figure 2 and reported below. Estimation statistics-based effects sizes and 95\% CIs were also calculated and are provided in Figure 2. Models with outliers included are shown in the Supplementary Materials; neither changes in the significance of models nor directionality of effects were observed with the inclusion of outliers. 


\section{Figure 2}

Violin- and box-plots (left) and paired mean difference estimates plots (right) show differences in the means of the temporal parameters between microstates.

a.
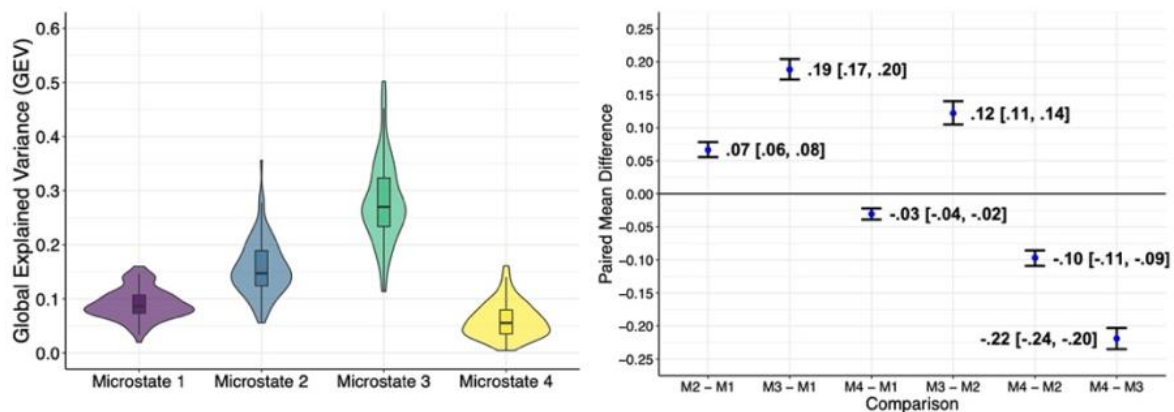

b.
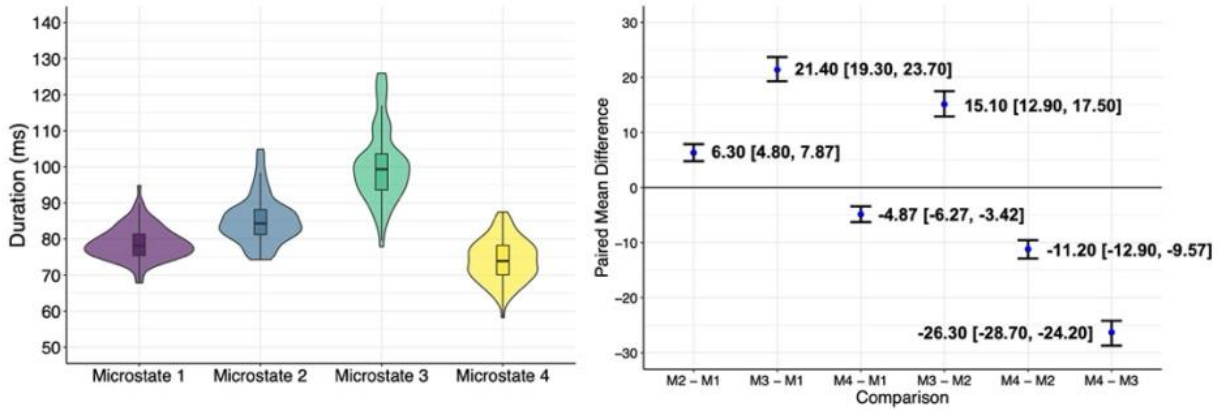

c.
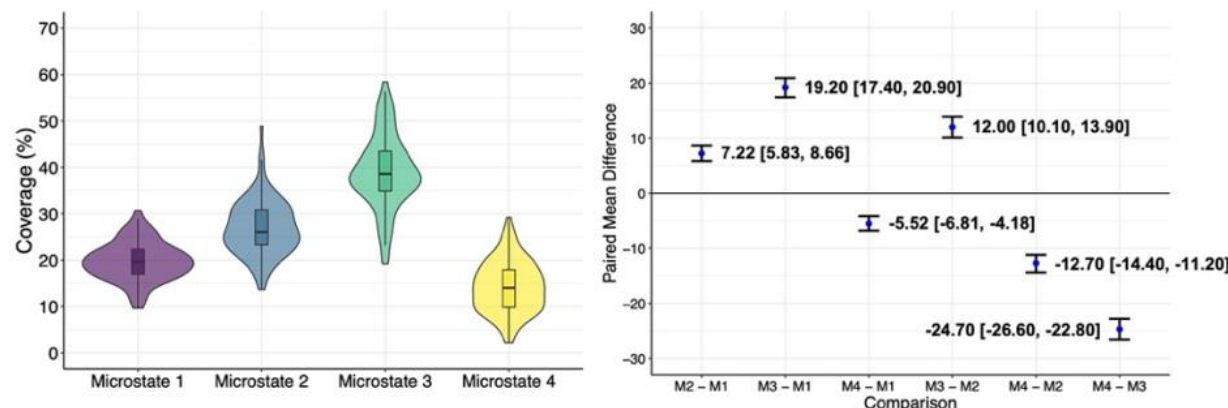

d.
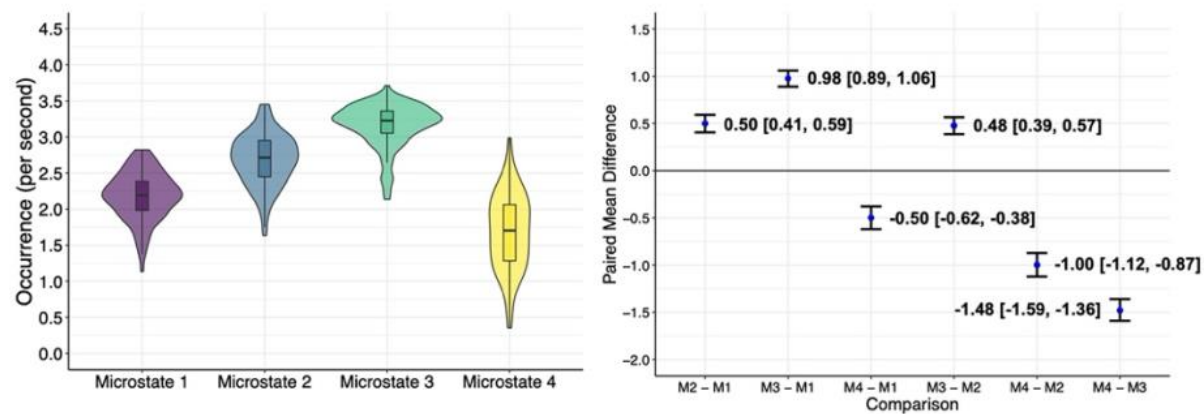

Note. All post-hoc combinations of ANOVA comparisons were significant at $p<.0001$, Benjamini-Hochberg-corrected for six multiple comparisons. Vertical bars in the paired mean difference estimates plots represent the $95 \%$ confidence interval of the paired mean difference estimates; numerical values are presented as the paired mean difference value $[95 \%$ confidence interval]. M1 = Microstate 1; M2 = Microstate 2; M3 = Microstate 3; M4 = Microstate 4. 


\subsubsection{GEV}

There was a significant difference in the mean GEV between microstates, $F(1.78,182.03)$ $=295.16, p<.0001, \eta^{2}{ }_{g}=0.74$. Planned post-hoc contrasts revealed that microstate $1 \mathrm{GEV}$ was lower than microstate $2 \mathrm{GEV}, t(102)=-11.24, p<.0001$, and microstate $3 \mathrm{GEV}, t(102)=-21.13$, $p<.0001$, but higher than microstate $4 \mathrm{GEV}, t(102)=7.34, p<.0001$. Microstate $2 \mathrm{GEV}$ was lower than microstate $3 \mathrm{GEV}, t(102)=-11.05, p<.0001$, but higher than microstate $4 \mathrm{GEV}, t(102)$ $=16.03$, corrected $p<.0001$. Microstate $3 \mathrm{GEV}$ was higher than microstate $4 \mathrm{GEV}, t(102)=22.72$, corrected $p<.0001$.

\subsubsection{Duration}

There was a significant difference in the mean duration between microstates, $F(1.99$, 199.48) $=219.72, p<.0001, \eta^{2} g=0.66$. Planned post-hoc contrasts revealed that microstate 1 duration was lower than microstate 2 duration, $t(100)=-8.09, p<.0001$, and microstate 3 duration, $t(100)=-17.71, p<.0001$, but higher than microstate 4 duration, $t(100)=6.91, p<.0001$. Microstate 2 duration was lower than microstate 3 duration, $t(100)=-10.56, p<.0001$, but higher than microstate 4 duration, $t(100)=12.65, p<.0001$. Microstate 3 duration was higher than microstate 4 duration, $t(100)=19.95, p<.0001$.

\subsubsection{Coverage}

There was a significant difference in the mean coverage between microstates, $F(2.26$, $230.88)=241.56, p<.0001, \eta^{2}{ }_{g}=0.70$. Planned post-hoc contrasts revealed that microstate 1 coverage was lower than microstate 2 coverage, $t(102)=-9.62, p<.0001$, and microstate 3 coverage, $t(102)=-18.85, p<.0001$, but higher than microstate 4 coverage, $t(102)=7.98, p<$ 
.0001 . Microstate 2 coverage was lower than microstate 3 coverage, $t(102)=-9.86, p<.0001$, but higher than microstate 4 coverage, $t(102)=14.72, p<.0001$. Microstate 3 coverage was higher than microstate 4 coverage, $t(102)=21.21, p<.0001$.

\subsubsection{Occurrence}

There was a significant difference in the mean occurrence between microstates, $F(2.48$, 253.13) $=234.49, p<.0001, \eta^{2}{ }_{g}=0.67$. Planned post-hoc contrasts revealed that microstate 1 occurrence was lower than microstate 2 occurrence, $t(102)=-10.28, p<.0001$, and microstate 3 occurrence, $t(102)=-20.99, p<.0001$, but higher than microstate 4 occurrence, $t(102)=8.13, p<$ .0001 . Microstate 2 occurrence was lower than microstate 3 occurrence, $t(102)=-9.12, p<.0001$, but higher than microstate 4 occurrence, $t(102)=14.49, p<.0001$. Microstate 3 occurrence was higher than microstate 4 occurrence, $t(102)=21.01, p<.0001$.

\subsection{Age and sex effects of each microstate's temporal parameters}

The extent to which each microstate's four temporal parameters could be predicted by age, sex, and age by sex interaction was examined using multiple linear regression models, BenjaminiHochberg-adjusted for four comparisons at the level of the overall model. For interpretability, age values for Johnson-Neyman intervals are reported in years and only within the range of observed values in the sample. The results of models with outliers excluded are summarized in Table 3 and described below. Models with outliers included are presented in Supplementary Table 2; one model (microstate 3 duration) became nonsignificant with the inclusion of outliers. 
Table 3

Regressions examining age and sex effects of each microstate's temporal parameters.

\begin{tabular}{|c|c|c|c|c|c|c|c|c|c|c|c|c|c|c|c|c|}
\hline \multirow[b]{2}{*}{ Variable } & \multicolumn{4}{|c|}{ GEV } & \multicolumn{4}{|c|}{ Duration } & \multicolumn{4}{|c|}{ Coverage } & \multicolumn{4}{|c|}{ Occurrence } \\
\hline & $B$ & $S E B$ & $\beta$ & $p$ & $B$ & $S E B$ & $\beta$ & $p$ & $B$ & $S E B$ & $\beta$ & $p$ & $B$ & $S E B$ & $\beta$ & $p$ \\
\hline Microstate 1 & \multicolumn{4}{|c|}{$F(3,99)=2.13, R^{2}=.03, p=.17$} & \multicolumn{4}{|c|}{$F(3,98)=0.99, R^{2}=-.0002, p=.40$} & \multicolumn{4}{|c|}{$F(3,99)=1.92, R^{2}=.03, p=.17$} & \multicolumn{4}{|c|}{$F(3,98)=3.34, R^{2}=.06, p=.09$} \\
\hline (Intercept) & 0.09 & 0.003 & - & $<.001 * * *$ & 78.61 & 0.43 & - & $<.001 * * *$ & 19.66 & 0.41 & - & $<.001 * * *$ & 2.18 & 0.03 & - & $<.001 * * *$ \\
\hline Age & 0.003 & 0.003 & 0.10 & .30 & 0.48 & 0.41 & 0.12 & .24 & 0.31 & 0.38 & 0.08 & .42 & 0.02 & 0.03 & 0.07 & .46 \\
\hline Sex & 0.01 & 0.003 & 0.21 & $.03 *$ & 0.40 & 0.43 & 0.09 & .36 & 0.90 & 0.41 & 0.22 & $.03 *$ & 0.09 & 0.03 & 0.30 & $.003 * *$ \\
\hline Age $*$ Sex & -0.002 & 0.003 & -0.08 & .40 & 0.26 & 0.41 & 0.06 & .53 & -0.20 & 0.38 & -0.05 & .60 & -0.01 & 0.03 & -0.03 & .78 \\
\hline Microstate 2 & \multicolumn{4}{|c|}{$F(3,98)=0.41, R^{2}=-.02, p=.84$} & \multicolumn{4}{|c|}{$F(3,95)=0.47, R^{2}=-.02, p=.84$} & \multicolumn{4}{|c|}{$F(3,98)=0.28, R^{2}=-.02, p=.84$} & \multicolumn{4}{|c|}{$F(3,99)=1.01, R^{2}=.0003, p=.84$} \\
\hline (Intercept) & 0.16 & 0.005 & - & $<.001 * * *$ & 84.71 & 0.60 & - & $<.001 * * *$ & 26.79 & 0.56 & - & $<.001 * * *$ & 2.68 & 0.04 & - & $<.001 * * *$ \\
\hline Age & -0.002 & 0.004 & -0.04 & .73 & 0.62 & 0.55 & 0.12 & .26 & 0.02 & 0.53 & 0.004 & .97 & -0.03 & 0.03 & -0.09 & .37 \\
\hline Sex & 0.002 & 0.005 & 0.05 & .64 & -0.19 & 0.58 & -0.03 & .74 & 0.05 & 0.56 & 0.01 & .93 & -0.01 & 0.04 & -0.02 & .83 \\
\hline Age $*$ Sex & -0.004 & 0.004 & -0.09 & .38 & 0.002 & 0.55 & 0.0003 & .998 & -0.48 & 0.53 & -0.09 & .37 & -0.04 & 0.03 & -0.13 & .20 \\
\hline Microstate 3 & \multicolumn{4}{|c|}{$F(3,98)=1.82, R^{2}=.02, p=.15$} & \multicolumn{4}{|c|}{$F(3,97)=4.46, R^{2}=.09, p=.02 *$} & \multicolumn{4}{|c|}{$F(3,99)=2.97, R^{2}=.05, p=.07$} & \multicolumn{4}{|c|}{$F(3,92)=2.18, R^{2}=.04, p=.13$} \\
\hline (Intercept) & 0.28 & 0.01 & - & $<.001 * * *$ & 100.27 & 0.97 & - & $<.001 * * *$ & 39.24 & 0.77 & - & $<.001 * * *$ & 3.22 & 0.02 & - & $<.001 * * *$ \\
\hline Age & 0.01 & 0.01 & 0.08 & .41 & 1.40 & 0.91 & 0.15 & .13 & 0.61 & 0.72 & 0.08 & .41 & -0.03 & 0.02 & -0.14 & .17 \\
\hline Sex & -0.01 & 0.01 & -0.21 & $.04 *$ & -3.20 & 0.97 & -0.32 & $.001 * *$ & -2.18 & 0.77 & -0.27 & $.005 * *$ & -0.03 & 0.02 & -0.16 & .12 \\
\hline Age $*$ Sex & -0.01 & 0.01 & -0.07 & .46 & -0.19 & 0.91 & -0.02 & .83 & -0.39 & 0.72 & -0.05 & .59 & -0.02 & 0.02 & -0.12 & .23 \\
\hline Microstate 4 & \multicolumn{4}{|c|}{$F(3,97)=3.77, R^{2}=.08, p=.01^{*}$} & \multicolumn{4}{|c|}{$F(3,99)=3.70, R^{2}=.07, p=.01^{*}$} & \multicolumn{4}{|c|}{$F(3,99)=3.95, R^{2}=.08, p=.01^{*}$} & \multicolumn{4}{|c|}{$F(3,99)=3.84, R^{2}=.08, p=.01^{*}$} \\
\hline (Intercept) & 0.06 & 0.003 & - & $<.001 * * *$ & 74.08 & 0.57 & - & $<.001 * * *$ & 14.13 & 0.52 & - & $<.001 * * *$ & 1.67 & 0.05 & - & $<.001 * * *$ \\
\hline Age & -0.004 & 0.003 & -0.15 & .14 & -0.19 & 0.53 & -0.03 & .72 & -0.69 & 0.49 & -0.14 & .17 & -0.07 & 0.05 & -0.15 & .13 \\
\hline Sex & 0.01 & 0.003 & 0.20 & $.04 *$ & 0.67 & 0.57 & 0.11 & .24 & 1.04 & 0.52 & 0.19 & $.049 *$ & 0.11 & 0.05 & 0.20 & $.03 *$ \\
\hline Age $*$ Sex & 0.01 & 0.003 & 0.23 & $.02 *$ & 1.66 & 0.53 & 0.30 & $.002 * *$ & 1.31 & 0.49 & 0.26 & $.009 * *$ & 0.11 & 0.05 & 0.23 & $.02 *$ \\
\hline
\end{tabular}

Note. The denominator or error degrees of freedom for each model varies because observations flagged as outliers were removed (i.e., the sample size for each model varies). $R^{2}$ values were adjusted for three predictors. Full model $p$ values were Benjamini-Hochberg-corrected for four comparisons, but $p$ values at the predictor-level were not corrected: $* p<.05, * * p<.01, * * * p<.001$. 


\subsubsection{Microstates 1 and 2}

The complete set of predictors did not explain a significant proportion of the observed variation in either microstate 1 or $2 \mathrm{GEV}$, duration, coverage, or occurrence.

\subsubsection{Microstate 3}

The complete set of predictors explained a significant proportion of the observed variation in microstate 3 duration, with an effect size of Cohen's $f^{2}=.10$. The main effect of sex was significant, with males having a higher microstate 3 duration than females, $t(97)=-3.32, p=.001$, $95 \%$ CI $[-5.12,-1.29]$. Neither age, $t(97)=1.53, p=.13,95 \%$ CI $[-0.41,3.21]$, nor the age by sex interaction, $t(97)=-.21, p=.83,95 \%$ CI $[-2.01,1.62]$, was significant. The complete set of predictors did not explain a significant proportion of the observed variation in microstate $3 \mathrm{GEV}$, coverage, or occurrence.

\subsubsection{Microstate 4}

The complete set of predictors explained a significant proportion of the observed variation in all temporal parameters of microstate 4 :

For GEV, the effect size was Cohen's $f^{2}=.09$ (Figure 3a). The main effect of sex was significant, $t(97)=2.13, p=.04,95 \%$ CI $[0.0004,0.01]$, but the main effect of age was not, $t(97)$ $=-1.50, p=.14,95 \%$ CI $[-0.01,0.001]$. These main effects were qualified by the presence of a significant age by sex interaction, $t(97)=2.37, p=.02,95 \%$ CI [0.001, 0.01]. Post-hoc analyses

revealed the slope of age predicting microstate $4 \mathrm{GEV}$ was significant and negative for males, $t(97)$ $=-2.52, p=.01,95 \% \mathrm{CI}[-0.02,-0.002]$, but nonsignificant for females, $t(97)=0.68, p=.50,95 \%$ CI [-0.004, 0.01]. The Johnson-Neyman procedure revealed that the estimated slope for the 
prediction of microstate 4 GEV by sex was significantly $(p<.05)$ different from zero when age was $>6.31$ but $\leq 8.65$ years.

For duration, the effect size was Cohen's $f^{2}=.08$ (Figure 3b). Neither the main effects of age, $t(99)=-0.35, p=.72,95 \%$ CI [-1.25, 0.87], or sex, $t(99)=1.19, p=.24,95 \%$ CI [-0.45, 1.79], were significant. These main effects were qualified by the presence of a significant age by sex interaction, $t(99)=3.11, p=.002,95 \%$ CI $[0.60,2.72]$. Post-hoc analyses revealed the slope of age predicting microstate 4 duration was significant and negative for males, $t(99)=-2.24, p=.03$, $95 \%$ CI[-3.48, -0.21$]$, but significant and positive for females, $t(99)=2.16, p=.03,95 \%$ CI [0.12, 2.82]. The Johnson-Neyman procedure revealed that the estimated slope for the prediction of microstate 4 duration by sex was significantly $(p<.05)$ different from zero when age was either $\geq$ 4.51 but $<4.71$ or $>6.67$ but $\leq 8.65$ years.

For coverage, the effect size was Cohen's $f^{2}=.09$ (Figure 3c). The main effect of sex was significant, $t(99)=1.99, p=.049,95 \%$ CI [0.003, 2.08], but the main effect of age was not, $t(99)$ $=-1.40, p=.17,95 \%$ CI $[-1.67,0.29]$. These main effects were qualified by the presence of a significant age by sex interaction, $t(99)=2.66, p=.009,95 \% \mathrm{CI}[0.33,2.29]$. Post-hoc analyses revealed the slope of age predicting microstate 4 coverage was significant and negative for males, $t(99)=-2.63, p=.01,95 \%$ CI[-3.51, -0.49$]$, but nonsignificant for females, $t(99)=0.99, p=.33$, 95\% CI $[-0.63,1.87]$. The Johnson-Neyman procedure revealed that the estimated slope for the prediction of microstate 4 coverage by sex was significantly $(p<.05)$ different from zero when age was $>6.37$ but $\leq 8.65$ years. 
For occurrence, the effect size was Cohen's $f^{2}=.09$ (Figure 3d). The main effect of sex was significant, $t(99)=2.14, p=.03,95 \% \mathrm{CI}[0.01,0.21]$, but the main effect of age was not, $t(99)$ $=-1.54, p=.13,95 \% \mathrm{CI}[-0.17,0.02]$. These main effects were qualified by the presence of a significant age by sex interaction, $t(99)=2.39, p=.02,95 \%$ CI $[0.02,0.21]$. Post-hoc analyses revealed the slope of age predicting microstate 4 occurrence was significant and negative for males, $t(99)=-2.55, p=.01,95 \% \mathrm{CI}[-0.33,-0.04]$, but nonsignificant for females, $t(99)=0.67, p$ $=.51,95 \% \mathrm{CI}[-0.08,0.16]$. The Johnson-Neyman procedure revealed that the estimated slope for the prediction of microstate 4 occurrence by sex was significantly $(p<.05)$ different from zero when age was $>6.30$ but $\leq 8.65$ years. 


\section{Figure 3}

Statistically significant interactions between age and sex on the temporal parameters of microstate 4.

a.

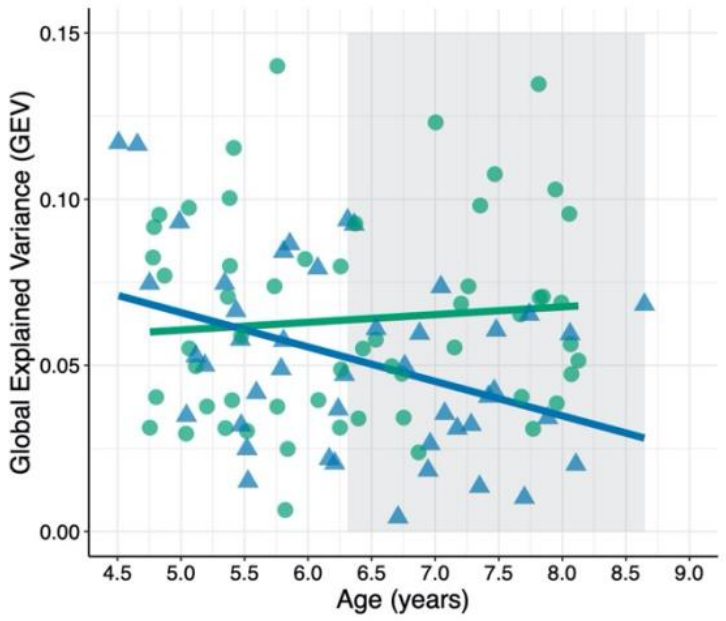

c.

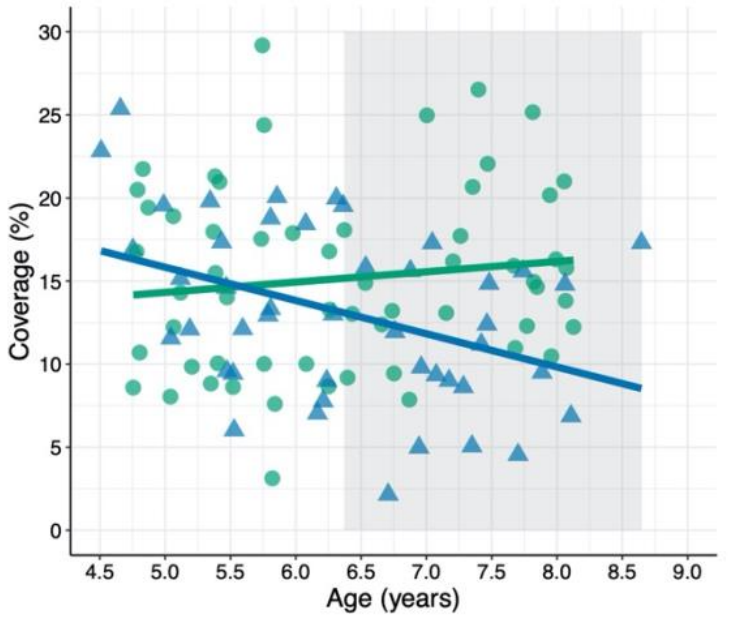

b.

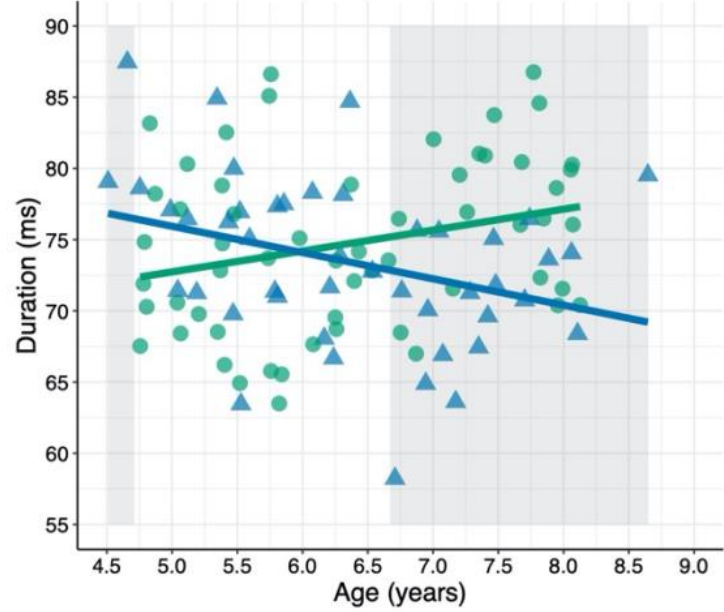

d.

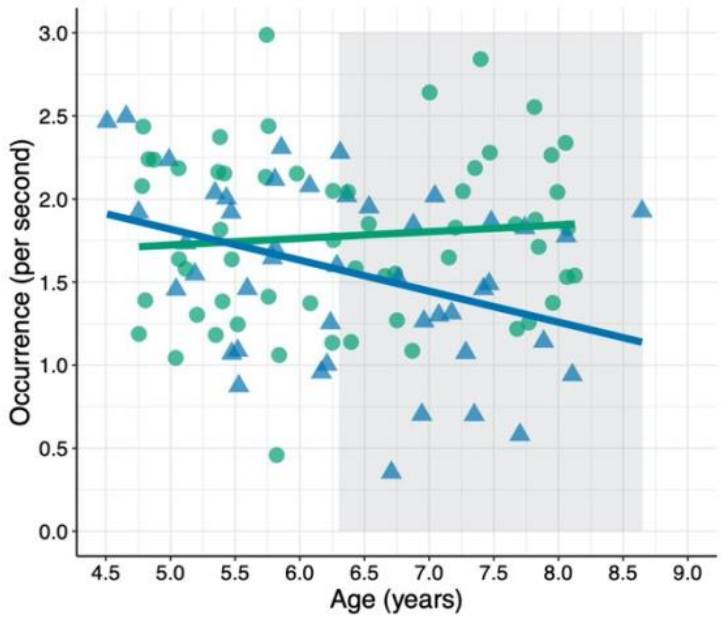

Note. Shaded regions represent Johnson-Neyman regions of significance. 


\subsection{Sources of Microstates}

The sources of each of the four microstates were estimated and thresholded to the solution points above the 95th percentile of activations across participants. The mean of the four thresholded source maps (Supplementary Figure 3) was subtracted from each of the source maps. The resulting cortical regions distinctly associated with each microstate are presented in Table 4 . Given the observed relationships between age and sex on the temporal parameters of microstates

3 and 4, their sources are overlayed on an MRI in Figure 4 (see Supplementary Figures 4-7 for the sources of all the microstates). 


\section{Table 4}

Neural sources of the four microstates.

\begin{tabular}{|c|c|}
\hline Microstate & Neural Sources \\
\hline Microstate 1 & $\begin{array}{l}\text { Left inferior temporal gyrus } \\
\text { Bilateral middle temporal gyri } \\
\text { Right superior temporal gyrus } \\
\text { Right anterior \& posterior cingulate cortex } \\
\text { Right inferior \& middle frontal gyri } \\
\text { Bilateral superior \& medial frontal gyri } \\
\text { Bilateral inferior \& middle occipital gyri } \\
\text { Left superior occipital gyrus } \\
\text { Bilateral fusiform gyri } \\
\text { Bilateral rectal gyrus } \\
\text { Right orbital gyrus } \\
\text { Right lingual gyrus } \\
\text { Bilateral cuneus } \\
\text { Right precuneus } \\
\text { Right superior parietal lobule } \\
\text { Bilateral precentral \& postcentral gyri } \\
\text { Bilateral paracentral lobules }\end{array}$ \\
\hline Microstate 2 & $\begin{array}{l}\text { Bilateral superior \& middle temporal gyri } \\
\text { Right inferior temporal gyrus } \\
\text { Left inferior frontal gyrus } \\
\text { Bilateral middle frontal gyri } \\
\text { Bilateral medial \& superior frontal gyri } \\
\text { Right superior \& inferior occipital gyri } \\
\text { Bilateral middle occipital gyri } \\
\text { Bilateral inferior parietal lobule } \\
\text { Left superior parietal lobule } \\
\text { Bilateral fusiform gyri } \\
\text { Left lingual gyrus } \\
\text { Bilateral cuneus \& precuneus } \\
\text { Right anterior cingulate cortex } \\
\text { Left supramarginal gyrus } \\
\text { Right postcentral gyrus }\end{array}$ \\
\hline Microstate 3 & $\begin{array}{l}\text { Bilateral inferior \& middle temporal gyri } \\
\text { Right superior temporal gyrus } \\
\text { Bilateral parahippocampal gyri } \\
\text { Bilateral fusiform gyri } \\
\text { Bilateral lingual gyri } \\
\text { Bilateral inferior \& middle occipital gyri } \\
\text { Bilateral supramarginal gyri } \\
\text { Right inferior frontal gyrus } \\
\text { Bilateral middle, medial, \& superior frontal gyri } \\
\text { Bilateral cuneus \& precuneus } \\
\text { Bilateral inferior and superior parietal lobules }\end{array}$ \\
\hline Microstate 4 & $\begin{array}{l}\text { Bilateral superior temporal gyri } \\
\text { Bilateral insula } \\
\text { Bilateral inferior, middle, medial, \& superior frontal gyri } \\
\text { Bilateral cuneus \& precuneus } \\
\text { Bilateral posterior cingulate cortex } \\
\text { Bilateral inferior \& superior parietal lobules } \\
\text { Bilateral supramarginal gyri } \\
\text { Bilateral precentral \& postcentral gyri } \\
\text { Bilateral paracentral lobules }\end{array}$ \\
\hline
\end{tabular}

Note. Neural sources were determined visually with the assistance of the whereami? function and Talairach-Tournoux Atlas in AFNI (Cox, 1996). Sources in the cerebellum and deep brain (i.e., subcortical) structures were ignored due to the lack of established validity in measuring these sources with EEG. 


\section{Figure 4}

Neural sources of Microstates 3 and 4.

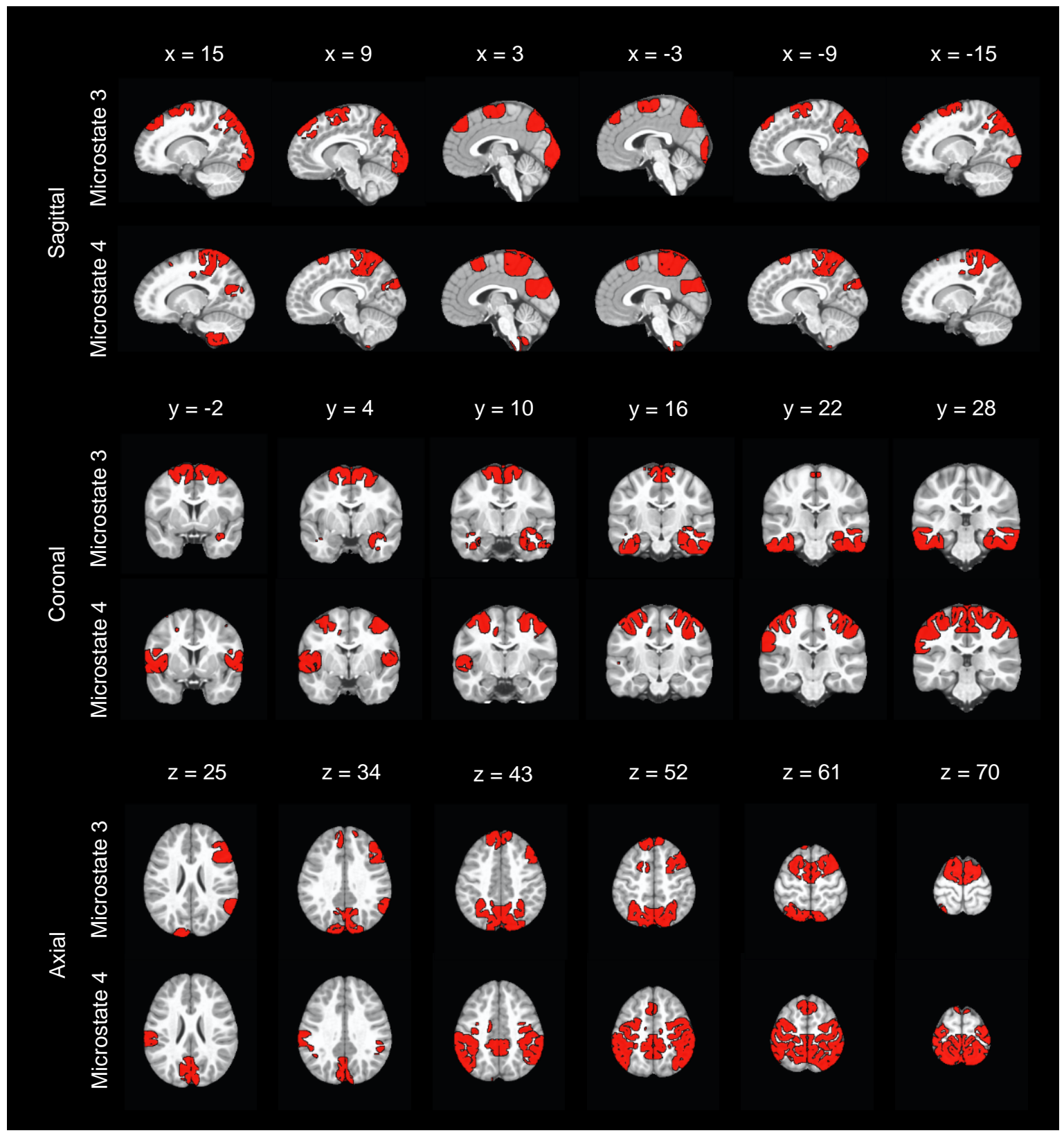

Note. Sagittal slices (x plane) are presented as left (positive coordinates) to right (negative coordinates) parts of the brain. Coronal slices (y plane) are presented as anterior (negative coordinates) to posterior (positive coordinates) parts of the brain. Axial slices ( $\mathrm{z}$ plane) are presented as inferior to superior parts of the brain. 


\section{Discussion}

The current study conducted a novel investigation of EEG microstates in a large sample of 4-8-year-old children using a data-driven method. As reported previously, four canonical microstates were replicated. Microstate 3 (canonical C) had the largest temporal parameters while microstate 4 (canonical D) had the smallest values relative to the other microstates (Koenig et al., 2002; Tomescu et al., 2018). In line with Tomescu et al. (2018), the temporal parameters of microstates 3 and 4 showed relationships with age and sex while those of microstates 1 (canonical A) and 2 (canonical B) did not. Microstate 3 duration was more prominent for males compared to females, and microstate 4 showed age by sex interactions for all its temporal parameters. The values of microstate $4 \mathrm{GEV}$, coverage, and occurrence decreased with increasing age for males but did not change with age for females. The values of microstate 4 duration decreased with age for males but increased with age for females. Post-hoc analyses revealed that the differences in the slopes of age between sexes changed from non-significant to significant at approximately 6.5 years across all microstate 4 models. Further, EEG source localization suggested observed effects potentially relate to attention- and cognitive control-related networks.

\subsection{Four microstate topographies are present in young children}

A four-microstate solution was the best fit for the EEG data in our sample of children, mirroring previous reports of four microstates in adults. These microstates each had a similar scalp potential topography as those previously reported in adults (see Figure 3 in Michel \& Koenig, 2018), suggesting that despite ongoing brain development in children, a similar global organization of scalp topographies exists between children and adults. This also suggests that, rather than large topographic changes in patterns of scalp potentials, it is their temporal parameters that change in 
children with development. This pattern of results parallels fMRI studies showing globally similar RSNs between children and adults, with differences largely seen in within- and between-network patterns of connectivity rather than network structure (Bie et al., 2012; Muetzel et al., 2016; Supekar et al., 2009).

\subsection{Source localization of microstates suggests relationships with fMRI RSNs and provides insight into age and sex relationships with temporal parameters}

Source localization indicated that the neural generators for microstate 3 partially overlap with regions of the dorsal frontoparietal network (D-FPN; Uddin et al., 2019). Commonly referred to as the "dorsal attention network," the D-FPN is broadly involved in visuospatial attention and is thought to be responsible for goal-directed, top-down processing (Farrant \& Uddin, 2015; Uddin et al., 2019; Yeo et al., 2011). Microstate 3 also revealed sources that partially overlap with regions of the lateral frontoparietal network (L-FPN), broadly involved in goal-directed control processes such as executive functions (Uddin et al., 2019). One subsystem within the L-FPN has been shown to preferentially connect to regions of the D-FPN and may be involved in the regulation of visuospatial attention and working memory performance (Dixon et al., 2018; Murphy et al., 2020). Portions of the D-FPN and L-FPN may underly the same microstate and support attentional orienting and control processes, which undergo a critical period of development during early childhood (Anderson, 2002; Rueda et al., 2005). As such, the observation that males had larger microstate 3 durations than females may signal a maturational difference in the development of attention and/or control systems between sexes. For example, at rest, males may spend more time in microstate 3 than females. However, the meaning of lower versus higher values of a particular microstate's duration is not well understood; spending more or less time in certain states may be 
advantageous or disadvantageous. Taken together, more research is needed to better understand the underlying sources of microstate 3 , whether these sources are different in children compared to adults, and the relationships between its temporal parameters and age and sex.

Source localization indicated that the neural generators for microstate 4 partially overlap with regions of the M-CIN, another control network broadly responsible for identifying salient information in line with current goals as well as for switching between the medial frontoparietal network (i.e., the default mode network or M-FPN) and the L-FPN (Goulden et al., 2014; Uddin et al., 2019). The M-CIN also includes the previously characterized "ventral attention" and "cingulo-opercular" networks, which are involved in directing attention to the spatial locations of salient stimuli and in set-maintenance activities, respectively (Corbetta \& Shulman, 2002; Dosenbach et al., 2008; Uddin et al., 2019). The observation that the values of microstate 4 GEV, duration, coverage, and occurrence decreased with age for males but not females, except for duration, which increased with age for females, may indicate unique developmental trajectories in the activity of the M-CIN between 4- to 8-year-old males and females. In line with this hypothesis, the 4- to 8-year-old period is characterized by the rapid development of executive function and moderate-to-strong correlations between age and performance on executive function measures (Zelazo et al., 2008; Zelazo \& Carlson, 2012). Sex differences in some executive functions may also exist during the same developmental period, but findings are mixed, with females outperforming males in some studies (Berlin \& Bohlin, 2002; Carlson \& Moses, 2001) and males outperforming females in others (Brocki \& Bohlin, 2004). As such, the M-CIN may be undergoing sex-specific reorganization, particularly in the approximately 6.5- to 8-year-old period when the difference in the temporal parameters between sexes is most pronounced, paralleled by changes in 
executive function. While the plotted slopes of males and females diverged with increasing age in the current sample, it is also possible that a nonlinear pattern of development may be present over a longer period as network connections continue to be refined and shaped by experience. Further longitudinal research is necessary to address this question.

Lastly, as suggested by Tomescu et al. (2018), it is possible that microstates 1 and 2 did not show relationships with age and sex because they may represent RSNs involved in basic sensory functions that have robust functional organizations early in life. Britz et al. (2010) and Custo et al. (2017) both found that microstates 1 and 2 were related to networks responsible for auditory (i.e., pericentral network) and visual processing (i.e., occipital network), respectively. However, clear network-specific relationships were not evident in our source localization results. Since previous studies have only been performed with adults, it is plausible that the sources underlying microstates 1 and 2 found in the current study reflect ongoing patterns of neurobiological development and/or developmentally specific patterns of network relationships in young children reflecting current task demands (i.e., sit still with eyes closed). However, the present study cannot make this functional distinction, and future longitudinal work will be necessary.

\subsection{Overlap of sources may reflect network flexibility and hub-like transition states}

In addition to a developmental explanation, the overlap of sources and hypothesized underlying networks between microstates in the current study may reflect global brain dynamics. For example, using fMRI in adults, the M-CIN has been shown to have a stable yet highly flexible organization; functional interactions of this network are among the most spatially varied in the 
brain (Chen et al., 2016). Furthermore, this network may be a hub for facilitating flexible interactions across networks and has been shown to predict individual differences in cognitive flexibility (Chen et al., 2016). Another example of network flexibility comes from studies of the M-FPN, which have demonstrated its regions to dynamically switch community memberships, adapt a global configuration, and have a critical role in higher-order cognitive processing (Vatansever et al., 2015). As a result, the current source localization findings suggest that microstate analyses may similarly reflect dynamic patterns of changing connectivity between brain regions but at a finer temporal resolution. Additional studies should further investigate this possibility to determine if microstates could be used to study developmental changes in dynamic network connectivity.

Our findings also show that some sources (e.g., precuneus) are present in all microstates and may be hubs that support multiple brain networks. Although our microstate analysis backfitting procedure accounted for all the data, the first clustering stage was performed only on GFP peaks, failing to capture topographies during periods of microstate transitions. While these periods have lower GFP and signal-to-noise, they have been shown to have complex dynamics missed by routine microstate analysis (Shaw et al., 2019). It is possible that these periods reflect hub-like transition states whose sources leak into all microstates. Supporting this hypothesis, precision dynamical mapping of fMRI data at the single participant-level has revealed hub-like transition states represented by all RSNs equally (Saggar et al., 2021). Investigation of transition periods may provide a fuller understanding of the spatiotemporal dynamics captured by microstate analysis. 


\subsection{Strengths, limitations, and future directions}

Our study, while cross-sectional, is the first to include typical children under the age of six years in a data-driven microstate analysis of resting-state EEG. With our large sample of 4-8-yearold children, we had enough variation in age to assess continuous relationships between microstate parameters and age and their interactions with sex, rather than group-level statistics between individuals grouped by specific ages. Furthermore, this is the first study to assess the underlying neural sources of microstates in children, offering promise for the utility of EEG microstates in studying functional brain development.

The lack of microstate research in typical children led this study to be exploratory rather than hypothesis-driven, and the results of this study should be interpreted with caution until replicated. The meaning of low versus high values of microstate temporal parameters is relatively unknown. Future longitudinal studies investigating the relationships between microstates and behavioral, cognitive, and clinical measures are needed to further understand the significance of the current findings for normative and atypical development.

Although source localization provides insight into the network of brain regions generating each microstate, the spatial resolution of EEG is markedly lower than fMRI. Still, the temporal resolution with which we can capture the spatiotemporal dynamics of brain activity provides unique and advantageous information that can further our developmental understanding of rapidly changing functional brain networks in children. Nevertheless, future use of individual MRI scans and EEG coordinate locations and multimodal EEG-fMRI studies may better assess the spatial overlap between EEG and fMRI RSNs. 
The current study also has implications for understanding brain network development in children with psychopathology. The spatiotemporal dynamics of microstates are sensitive to psychopathology that emerges during early childhood, most notably autism spectrum disorder (ASD) and attention-deficit/hyperactivity disorder (ADHD). Interestingly, for example, prior research has identified differences in the temporal parameters of microstates $\mathrm{C}$ (resembling microstate 3) and D (resembling microstate 4) between children and adults with ASD or ADHD and typical controls (Das et al., 2021; D’Croz-Baron et al., 2019; Férat et al., 2021; Jia \& Yu, 2019; Nagabhushan Kalburgi et al., 2020; Takarae et al., 2022). Our findings of age- and sexrelated effects in the temporal parameters of these two microstates may be critical for understanding these observed differences in children with ASD and/or ADHD - which also have known sex differences in diagnosis - as well as in other forms of childhood psychopathology.

\subsection{Conclusion}

The current study reports novel age- and sex-related effects in the spatiotemporal dynamics of EEG microstates using a large sample of 4- to 8-year-old children. Matching previous reports in older samples, data-driven analyses indicated that a four-microstate solution best characterized resting-state EEG data at this young age. Further, using source localization techniques, we found support for attention- and control-related systems governing the topographies of age- and sexdependent microstates. As a result, the current study provides unique insights into children's functional brain development using the EEG microstates approach. 


\section{References}

Anderson, P. (2002). Assessment and Development of Executive Function (EF) During Childhood. Child Neuropsychology, 8(2), 71-82. https://doi.org/10.1076/chin.8.2.71.8724

Benjamini, Y., \& Hochberg, Y. (1995). Controlling the False Discovery Rate: A Practical and Powerful Approach to Multiple Testing. Journal of the Royal Statistical Society. Series B (Methodological), 57(1), 289-300.

Ben-Shachar, M. S. (2018). TBT: Reject and interpolate channels on a epoch by epoch basis (2.6.1) [Computer software]. https://doi.org/10.5281/zenodo.1241518

Berlin, L., \& Bohlin, G. (2002). Response Inhibition, Hyperactivity, and Conduct Problems Among Preschool Children. Journal of Clinical Child \& Adolescent Psychology, 31(2), 242-251. https://doi.org/10.1207/S15374424JCCP3102_09

Bernard, C. (2019). Changing the Way We Report, Interpret, and Discuss Our Results to Rebuild Trust in Our Research. ENeuro, 6(4), ENEURO.0259-19.2019. https://doi.org/10.1523/ENEURO.0259-19.2019

Bie, H. M. A. de, Boersma, M., Adriaanse, S., Veltman, D. J., Wink, A. M., Roosendaal, S. D., Barkhof, F., Stam, C. J., Oostrom, K. J., Waal, H. A. D. de, \& Sanz-Arigita, E. J. (2012). Resting-state networks in awake five- to eight-year old children. Human Brain Mapping, 33(5), 1189-1201. https://doi.org/10.1002/hbm.21280

Bréchet, L., Brunet, D., Birot, G., Gruetter, R., Michel, C. M., \& Jorge, J. (2019). Capturing the spatiotemporal dynamics of self-generated, task-initiated thoughts with EEG and fMRI. NeuroImage, 194, 82-92. https://doi.org/10.1016/j.neuroimage.2019.03.029 
Bréchet, L., Brunet, D., Perogamvros, L., Tononi, G., \& Michel, C. M. (2020). EEG microstates of dreams. Scientific Reports, 10(1), 17069. https://doi.org/10.1038/s41598-020-74075-z

Bréchet, L., Ziegler, D. A., Simon, A. J., Brunet, D., Gazzaley, A., \& Michel, C. M. (2021).

Reconfiguration of Electroencephalography Microstate Networks after Breath-Focused, Digital Meditation Training. Brain Connectivity, 11(2), 146-155. https://doi.org/10.1089/brain.2020.0848

Britz, J., Van De Ville, D., \& Michel, C. M. (2010). BOLD correlates of EEG topography reveal rapid resting-state network dynamics. NeuroImage, 52(4), 1162-1170. https://doi.org/10.1016/j.neuroimage.2010.02.052

Brocki, K. C., \& Bohlin, G. (2004). Executive Functions in Children Aged 6 to 13: A Dimensional and Developmental Study. Developmental Neuropsychology, 26(2), 571593. https://doi.org/10.1207/s15326942dn2602_3

Brown, T. T., \& Jernigan, T. L. (2012). Brain Development During the Preschool Years. Neuropsychology Review, 22(4), 313-333. https://doi.org/10.1007/s11065-012-9214-1

Brunet, D., Murray, M. M., \& Michel, C. M. (2011). Spatiotemporal Analysis of Multichannel EEG: CARTOOL. Computational Intelligence and Neuroscience, 2011, e813870. https://doi.org/10.1155/2011/813870

Carlson, S. M., \& Moses, L. J. (2001). Individual Differences in Inhibitory Control and Children's Theory of Mind. Child Development, 72(4), 1032-1053. https://doi.org/10.1111/1467-8624.00333

Chen, T., Cai, W., Ryali, S., Supekar, K., \& Menon, V. (2016). Distinct Global Brain Dynamics and Spatiotemporal Organization of the Salience Network. PLOS Biology, 14(6), e1002469. https://doi.org/10.1371/journal.pbio.1002469 
Claridge-Chang, A., \& Assam, P. N. (2016). Estimation statistics should replace significance testing. Nature Methods, 13(2), 108-109. https://doi.org/10.1038/nmeth.3729

Corbetta, M., \& Shulman, G. L. (2002). Control of goal-directed and stimulus-driven attention in the brain. Nature Reviews Neuroscience, 3(3), 201-215. https://doi.org/10.1038/nrn755

Cox, R. W. (1996). AFNI: Software for Analysis and Visualization of Functional Magnetic Resonance Neuroimages. Computers and Biomedical Research, 29(3), 162-173. https://doi.org/10.1006/cbmr.1996.0014

Custo, A., Van De Ville, D., Wells, W. M., Tomescu, M. I., Brunet, D., \& Michel, C. M. (2017). Electroencephalographic Resting-State Networks: Source Localization of Microstates. Brain Connectivity, 7(10), 671-682. https://doi.org/10.1089/brain.2016.0476

Das, S., Zomorrodi, R., Enticott, P., Kirkovski, M., Blumberger, D. M., Rajji, T. K., \& Desarkar, P. (2021). Atypical Resting State EEG Microstates in Autism: Preliminary Results. Biological Psychiatry, 89(9, Supplement), S347. https://doi.org/10.1016/j.biopsych.2021.02.865

D’Croz-Baron, D. F., Baker, M., Michel, C. M., \& Karp, T. (2019). EEG Microstates Analysis in Young Adults With Autism Spectrum Disorder During Resting-State. Frontiers in Human Neuroscience, 13. https://www.frontiersin.org/article/10.3389/fnhum.2019.00173

Delorme, A., \& Makeig, S. (2004). EEGLAB: An open source toolbox for analysis of single-trial EEG dynamics including independent component analysis. Journal of Neuroscience Methods, 134(1), 9-21. https://doi.org/10.1016/j.jneumeth.2003.10.009

Dixon, M. L., Vega, A. D. L., Mills, C., Andrews-Hanna, J., Spreng, R. N., Cole, M. W., \& Christoff, K. (2018). Heterogeneity within the frontoparietal control network and its 
relationship to the default and dorsal attention networks. Proceedings of the National Academy of Sciences, 115(7), E1598-E1607. https://doi.org/10.1073/pnas.1715766115

Dosenbach, N. U. F., Fair, D. A., Cohen, A. L., Schlaggar, B. L., \& Petersen, S. E. (2008). A dual-networks architecture of top-down control. Trends in Cognitive Sciences, 12(3), 99105. https://doi.org/10.1016/j.tics.2008.01.001

Farrant, K., \& Uddin, L. Q. (2015). Asymmetric development of dorsal and ventral attention networks in the human brain. Developmental Cognitive Neuroscience, 12, 165-174. https://doi.org/10.1016/j.den.2015.02.001

Férat, V., Arns, M., Deiber, M.-P., Hasler, R., Perroud, N., Michel, C. M., \& Ros, T. (2021). Electroencephalographic Microstates as Novel Functional Biomarkers for Adult Attention-Deficit/Hyperactivity Disorder. Biological Psychiatry: Cognitive Neuroscience and Neuroimaging. https://doi.org/10.1016/j.bpsc.2021.11.006

Goulden, N., Khusnulina, A., Davis, N. J., Bracewell, R. M., Bokde, A. L., McNulty, J. P., \& Mullins, P. G. (2014). The salience network is responsible for switching between the default mode network and the central executive network: Replication from DCM. NeuroImage, 99, 180-190. https://doi.org/10.1016/j.neuroimage.2014.05.052

Ho, J., Tumkaya, T., Aryal, S., Choi, H., \& Claridge-Chang, A. (2019). Moving beyond P values: Data analysis with estimation graphics. Nature Methods, 16(7), 565-566. https://doi.org/10.1038/s41592-019-0470-3

Jia, H., \& Yu, D. (2019). Aberrant Intrinsic Brain Activity in Patients with Autism Spectrum Disorder: Insights from EEG Microstates. Brain Topography, 32(2), 295-303. https://doi.org/10.1007/s10548-018-0685-0 
Johnson, M. H. (2011). Interactive Specialization: A domain-general framework for human functional brain development? Developmental Cognitive Neuroscience, 1(1), 7-21. https://doi.org/10.1016/j.dcn.2010.07.003

Johnson, P. O., \& Neyman, J. (1936). Tests of certain linear hypotheses and their application to some educational problems. Statistical Research Memoirs, 1, 57-93.

Khanna, A., Pascual-Leone, A., Michel, C. M., \& Farzan, F. (2015). Microstates in resting-state EEG: Current status and future directions. Neuroscience \& Biobehavioral Reviews, 49, 105-113. https://doi.org/10.1016/j.neubiorev.2014.12.010

Koenig, T., Prichep, L., Lehmann, D., Sosa, P. V., Braeker, E., Kleinlogel, H., Isenhart, R., \& John, E. R. (2002). Millisecond by Millisecond, Year by Year: Normative EEG Microstates and Developmental Stages. NeuroImage, 16(1), 41-48. https://doi.org/10.1006/nimg.2002.1070

Kraemer, H. C., \& Blasey, C. M. (2004). Centring in regression analyses: A strategy to prevent errors in statistical inference. International Journal of Methods in Psychiatric Research, 13(3), 141-151. https://doi.org/10.1002/mpr.170

Lee, T.-W., Girolami, M., \& Sejnowski, T. J. (1999). Independent Component Analysis Using an Extended Infomax Algorithm for Mixed Subgaussian and Supergaussian Sources. Neural Computation, 11(2), 417-441. https://doi.org/10.1162/089976699300016719

Lehmann, D. (2010). Multimodal analysis of resting state cortical activity: What does fMRI add to our knowledge of microstates in resting state EEG activity? NeuroImage, 52(4), 11731174. https://doi.org/10.1016/j.neuroimage.2010.05.033 
Lehmann, D., Ozaki, H., \& Pal, I. (1987). EEG alpha map series: Brain micro-states by spaceoriented adaptive segmentation. Electroencephalography and Clinical Neurophysiology, 67(3), 271-288. https://doi.org/10.1016/0013-4694(87)90025-3

Liu, J., Xu, J., Zou, G., He, Y., Zou, Q., \& Gao, J.-H. (2020). Reliability and Individual Specificity of EEG Microstate Characteristics. Brain Topography, 33(4), 438-449. https://doi.org/10.1007/s10548-020-00777-2

Long, X., Benischek, A., Dewey, D., \& Lebel, C. (2017). Age-related functional brain changes in young children. NeuroImage, 155, 322-330. https://doi.org/10.1016/j.neuroimage.2017.04.059

Michel, C. M., \& Brunet, D. (2019). EEG Source Imaging: A Practical Review of the Analysis Steps. Frontiers in Neurology, 10, 325. https://doi.org/10.3389/fneur.2019.00325

Michel, C. M., \& Koenig, T. (2018). EEG microstates as a tool for studying the temporal dynamics of whole-brain neuronal networks: A review. NeuroImage, 180, 577-593. https://doi.org/10.1016/j.neuroimage.2017.11.062

Muetzel, R. L., Blanken, L. M. E., Thijssen, S., Lugt, A. van der, Jaddoe, V. W. V., Verhulst, F. C., Tiemeier, H., \& White, T. (2016). Resting-state networks in 6-to-10 year old children. Human Brain Mapping, 37(12), 4286-4300. https://doi.org/10.1002/hbm.23309

Mullen, T. (2012). NITRC: CleanLine: Tool/Resource Info.

Mullen, T. R., Kothe, C. A. E., Chi, Y. M., Ojeda, A., Kerth, T., Makeig, S., Jung, T. P., \& Cauwenberghs, G. (2015). Real-time neuroimaging and cognitive monitoring using wearable dry EEG. IEEE Transactions on Biomedical Engineering, 62(11), 2553-2567. https://doi.org/10.1109/TBME.2015.2481482 
Murphy, A. C., Bertolero, M. A., Papadopoulos, L., Lydon-Staley, D. M., \& Bassett, D. S. (2020). Multimodal network dynamics underpinning working memory. Nature Communications, 11(1), 3035. https://doi.org/10.1038/s41467-020-15541-0

Nagabhushan Kalburgi, S., Whitten, A. P., Key, A. P., \& Bodfish, J. W. (2020). Children With Autism Produce a Unique Pattern of EEG Microstates During an Eyes Closed RestingState Condition. Frontiers in Human Neuroscience, 14, 288. https://doi.org/10.3389/fnhum.2020.00288

Pascual-Marqui, R. D., Michel, C. M., \& Lehmann, D. (1994). Low resolution electromagnetic tomography: A new method for localizing electrical activity in the brain. International Journal of Psychophysiology, 18(1), 49-65. https://doi.org/10.1016/01678760(84)90014-X

Pascual-Marqui, R. D., Michel, C. M., \& Lehmann, D. (1995). Segmentation of brain electrical activity into microstates: Model estimation and validation. IEEE Transactions on Biomedical Engineering, 42(7), 658-665. https://doi.org/10.1109/10.391164

Pion-Tonachini, L., Kreutz-Delgado, K., \& Makeig, S. (2019). ICLabel: An automated electroencephalographic independent component classifier, dataset, and website. NeuroImage, 198, 181-197. https://doi.org/10.1016/j.neuroimage.2019.05.026

Rousseeuw, P. J., \& Driessen, K. V. (1999). A Fast Algorithm for the Minimum Covariance Determinant Estimator. Technometrics, 41(3), 212-223. https://doi.org/10.1080/00401706.1999.10485670

Rueda, M. R., Rothbart, M. K., McCandliss, B. D., Saccomanno, L., \& Posner, M. I. (2005). Training, maturation, and genetic influences on the development of executive attention. 
Proceedings of the National Academy of Sciences, 102(41), 14931-14936.

https://doi.org/10.1073/pnas.0506897102

Saggar, M., Shine, J. M., Liégeois, R., Dosenbach, N. U. F., \& Fair, D. (2021). Precision dynamical mapping using topological data analysis reveals a unique hub-like transition state at rest (p. 2021.08.05.455149). https://doi.org/10.1101/2021.08.05.455149

Shaw, S. B., Dhindsa, K., Reilly, J. P., \& Becker, S. (2019). Capturing the Forest but Missing the Trees: Microstates Inadequate for Characterizing Shorter-Scale EEG Dynamics. Neural Computation, 31(11), 2177-2211.https://doi.org/10.1162/neco_a_01229

Supekar, K., Musen, M., \& Menon, V. (2009). Development of Large-Scale Functional Brain Networks in Children. PLOS Biology, 7(7), e1000157. https://doi.org/10.1371/journal.pbio.1000157

Takarae, Y., Zanesco, A., Keehn, B., Chukoskie, L., Müller, R.-A., \& Townsend, J. (2022). EEG microstates suggest atypical resting-state network activity in high-functioning children and adolescents with autism spectrum development. Developmental Science, e13231. https://doi.org/10.1111/desc.13231

Tomescu, M. I., Rihs, T. A., Rochas, V., Hardmeier, M., Britz, J., Allali, G., Fuhr, P., Eliez, S., \& Michel, C. M. (2018). From swing to cane: Sex differences of EEG resting-state temporal patterns during maturation and aging. Developmental Cognitive Neuroscience, 31, 58-66. https://doi.org/10.1016/j.den.2018.04.011

Uddin, L. Q., Yeo, B. T. T., \& Spreng, R. N. (2019). Towards a universal taxonomy of macroscale functional human brain networks. Brain Topography, 32(6), 926-942. https://doi.org/10.1007/s10548-019-00744-6 
Vatansever, D., Menon, D. K., Manktelow, A. E., Sahakian, B. J., \& Stamatakis, E. A. (2015). Default Mode Dynamics for Global Functional Integration. Journal of Neuroscience, 35(46), 15254-15262. https://doi.org/10.1523/JNEUROSCI.2135-15.2015

Ville, D. V. D., Britz, J., \& Michel, C. M. (2010). EEG microstate sequences in healthy humans at rest reveal scale-free dynamics. Proceedings of the National Academy of Sciences, 107(42), 18179-18184. https://doi.org/10.1073/pnas.1007841107

Yeo, B. T., Krienen, F. M., Sepulcre, J., Sabuncu, M. R., Lashkari, D., Hollinshead, M., Roffman, J. L., Smoller, J. W., Zöllei, L., Polimeni, J. R., Fischl, B., Liu, H., \& Buckner, R. L. (2011). The organization of the human cerebral cortex estimated by intrinsic functional connectivity. Journal of Neurophysiology, 106(3), 1125-1165. https://doi.org/10.1152/jn.00338.2011

Zelazo, P. D., \& Carlson, S. M. (2012). Hot and Cool Executive Function in Childhood and Adolescence: Development and Plasticity. Child Development Perspectives, 6(4), 354360. https://doi.org/10.1111/j.1750-8606.2012.00246.x

Zelazo, P. D., Carlson, S. M., \& Kesek, A. (2008). The development of executive function in childhood. In Handbook of developmental cognitive neuroscience, 2nd ed (pp. 553-574). MIT Press. 


\section{Appendix A. Supplementary materials}

For the Methods section:

Inclusion and exclusion criteria

Participants were part of a larger study investigating reward processing in early emerging risk for depression. Parents were required to be the child's biological mother and primary caregiver, and to have lived with the child for the last six months. Children were required to be 4- to 7 years old at the date of their first visit. Children were excluded if (1) they were born premature (<35 weeks of gestation), (2) their biological mother drank alcohol, used illicit drugs, or smoked during pregnancy, (3) they ever experienced seizures, loss of consciousness, brain swelling, or hospitalization due to head trauma, (4) they were diagnosed with Hydrocephalus, Hypoxic-Ischemic Encephalopathy, Cerebral Palsy, a brain tumor, a genetic disorder, hearing or vision impairment, a chronic illness, Attention-Deficit/Hyperactivity Disorder, or a Pervasive Developmental Disorder (PDD), (5) they had delays in speech or language or other developmental delays, (6) they had a medical problem or illness that caused behavioral concerns, or (7) they took medications for mood, anxiety, or attention difficulties. Caregivers were also asked about their child's depressive symptoms using the short version of the Preschool Feelings Checklist (PFC-Short; Luby et al., 2004). Due to the primary aims of the overarching study, children with low ( $\leq 1$ PFC items endorsed) or high ( $\geq 3$ PFC items endorsed) levels of depressive symptoms were included while children with a moderate (2 PFC items endorsed) level were excluded. 
Statistical analyses assessing differences between the age, number of males and females, and depressive symptom scores of the 140 eligible children who participated at a time when EEG was part of the study protocol (full sample) and the 103 participants who provided adequate EEG data (sub-sample)

(1) Age: A Wilcoxon Rank Sum test showed that there was no significant difference in children's age between the full sample and the sub-sample ( $W=6746.5, p=.70)$. A Wilcoxon Rank Sum test was performed because the ages of the full sample ( $W=0.94, p<$ $.001)$ and sub-sample $(W=0.95, p<.001)$ were not normally distributed according to quantile-quantile plots.

(2) Depressive symptoms: A Wilcoxon Rank Sum test showed that there was no significant difference in children's depressive symptoms between the full sample and the sub-sample ( $W$ $=6746.5, p=.70)$. A Wilcoxon Rank Sum test was performed because the depressive symptoms of the full sample $(W=0.97, p=.003)$ and sub-sample $(W=0.96, p=.006)$ were not normally distributed according to quantile-quantile plots. Children's depressive symptoms are represented as the unstandardized residual values from a regression model in which the Beck Depression Inventory, 2nd edition (BDI-II; Beck et al., 1996) was entered as the independent variable and the Preschool Feelings Checklist, Scale Version (PFC-Scale; Luby et al., 2012) was entered as the dependent variable (these measures were collected at each study visit as part of the overarching study). This procedure reduced the inflation of children's depressive symptoms by maternal depressive symptoms. The sample size for this analysis was reduced to 131 because nine participants did not have a PFC-Scale and/or BDI2 score. 
(3) Sex: A two-sample test for the equality of proportions showed that the proportion of males and females between the full sample (.53) and sub-sample (.56) were not significantly different $(X$-squared $=0.16, p=.69)$.

Note. Wilcoxon Rank Sum tests may not be appropriate for the age and depressive symptoms analyses since the samples were not independent (i.e., all sub-sample participants were part of the full sample). 


\section{Detailed EEG preprocessing steps}

Twenty-four channels located on the outer ring of the EEG sensor net were removed because they contained a large amount of artifact given their location near the base of the skull or on the neck or face. Data were downsampled to $250 \mathrm{~Hz}$ and filtered in two passes using zerophase, non-causal, finite impulse response filters: A low pass filter to remove frequencies above $40 \mathrm{~Hz}$, followed by a high pass filter to remove frequencies below $1 \mathrm{~Hz}$. Although a $40 \mathrm{~Hz}$ low pass filter was applied, $60 \mathrm{~Hz}$ electrical line noise was still present for some participants and subsequently attenuated for all participants using the CleanLine plugin (Mullen, 2012). Bad channels were removed using the following criteria: if the channel 1) was flat for more than five seconds, 2) contained more than four standard deviations of line noise relative to its signal (based on the total channel population), or 3) correlated at less than .8 to an estimate based on nearby channels. Artifact Subspace Reconstruction (ASR) was implemented to remove portions of data containing artifacts (Mullen et al., 2015). For ASR, the burst criterion or maximum acceptable 0.5 second window standard deviation was set conservatively to 20 (Chang et al., 2018). Additional periods of data were marked as bad if a channel's power exceeded seven standard deviations; periods were removed only if more than $25 \%$ of channels during a given period were marked as bad.

Extended infomax independent component analysis (ICA) was used to decompose the data into independent components (Lee et al., 1999). Given the large number of channels and a relatively small number of data points, principal component analysis (PCA) was applied to generate a subspace of 30 components before performing ICA. This number of components was chosen based on visual inspection of ICA decompositions with varying components for a subset of participants as well as a rule-of-thumb formula endorsed by the Swartz Center for 
Computational Neuroscience (San Diego, CA) to estimate the minimum length of data for ICA training (20 to 30 timepoints per channel squared). The ICA matrix was copied over to the fulllength data (i.e., the data just before ASR removed portions containing artifacts). The ICLabel plugin, an independent component classifier trained on crowdsourced labels (Pion-Tonachini et al., 2019), removed independent components with a probability greater than .7 of being eye or muscle artifact. We removed independent components related to eye and muscle artifacts because they were the most prominent as observed with visual inspection of the raw data and independent components.

Data were segmented into nonoverlapping one-second epochs and removed based on the following criteria using the TBT plugin (Ben-Shachar, 2018): At least 10 channels with 1) amplitudes greater than 100 microvolts $(\mu \mathrm{V})$ or less than $-100 \mu \mathrm{V}$, or 2) joint probabilities (i.e., probabilities of activity) above 3 standard deviations for local or global thresholds. Together, these criteria are an effective combination for removing high-amplitude noise (Delorme et al., 2007), have previously been used with developmental data (Piazza et al., 2016), and are implemented in a published standardized preprocessing pipeline (Gabard-Durnam et al., 2018). If less than 10 channels met rejection criteria, the epoch was not removed, but the channels were interpolated for that epoch only. Lastly, channels removed previously were interpolated using spherical splines and all channels were re-referenced to the average. Preprocessed data were assessed for quality and participants who did not pass quality control were removed from further analyses (see below). After all preprocessing and the removal of five participants whose data did not pass quality control, the minimum amount of data across 103 participants was 145 seconds. To reduce the potential effects of varying data lengths across participants on further analyses, 
data for all participants were trimmed to their first 145 second, which exceeds the previously published two-minute mark for reliability of microstate analysis (Liu et al., 2020). 


\section{Quality control of EEG data}

Liu et al. (2020) demonstrated for microstate analysis that resting state EEG data with a duration greater than two minutes has moderate to high reliability and individual specificity; of the 108 participants with EEG data, five with less than two minutes of clean data were excluded from further analyses.

There were no participants with an excessive number of bad channels removed (i.e., excessive defined as more than $\sim 15 \%$ or $\sim 16$ channels whereas the maximum in the current sample was $12.5 \%$ or 13 channels) and visual inspection of bad channel plots revealed no clusters of bad channels (i.e., a cluster defined as a channel surrounded by all bad channels with no immediate neighboring good channel). Channel spectra plots were examined and looked appropriate for all participants.

Since Independent Component Analysis (ICA) was applied to data cleaned by Artifact Subspace Reconstruction (ASR), each participant's file length after ASR was examined to ensure there were no files with less than approximately 60 seconds of data after ASR's removal of data; otherwise, ICA would produce poor or unreliable results due to a lack of enough data points for training. All participants had at least 59.56 seconds of data post-ASR. Independent component plots were examined and looked appropriate for all participants, and those removed by the ICLabel plugin as being eye or muscle artifact with a probability greater than .7 were visually confirmed as being eye or muscle artifact. Retained variance in the data was above $50.17 \%$ for all participants after removal of independent components related to eye or muscle artifact. However, retained variance may be a slightly misleading metric since PCA was performed before ICA (i.e., some variance was already lost before ICA was performed). 


\section{Supplementary Table 1}

Descriptive statistics of EEG data quality metrics.

\begin{tabular}{lccccc}
\hline & $\begin{array}{c}\text { Number } \\
\text { File Length in } \\
\text { After Preprocessing } \\
\text { (Percent) of } \\
\text { Channels } \\
\text { Removed and } \\
\text { Interpolated }\end{array}$ & $\begin{array}{c}\text { File Length in } \\
\text { Seconds (Percent) } \\
\text { After ASR }\end{array}$ & $\begin{array}{c}\text { Number } \\
\text { (Percent) of } \\
\text { Independent } \\
\text { Components } \\
\text { Rejected }\end{array}$ & $\begin{array}{c}\text { Percent } \\
\text { Retaince } \\
\text { After } \\
\text { Removal of } \\
\text { Independent } \\
\text { Components }\end{array}$ \\
\hline Mean & $214.31(89.30 \%)$ & $4.20(4.04 \%)$ & $193.86(80.78 \%)$ & $2.98(9.93 \%)$ & $79.19 \%$ \\
SD & $16.61(6.92 \%)$ & $3.16(3.04 \%)$ & $34.30(14.29 \%)$ & $1.32(4.4 \%)$ & $11.08 \%$ \\
Minimum & $145(60.42 \%)$ & $0(0 \%)$ & $59.56(24.82 \%)$ & $0(0 \%)$ & $50.17 \%$ \\
\end{tabular}

Note. Descriptive statistics are for the 103 clean EEG files. ASR $=$ Artifact Subspace Reconstruction. 
Versions of EEG preprocessing tools

- MATLAB - R2019b

- EEGLAB - 2021.0

- firfilt EEGLAB plugin - 2.4

- CleanLine EEGLAB plugin - 2.00

- Clean Rawdata EEGLAB plugin - 2.3

- ICLabel EEGLAB plugin - 1.3

- TBT EEGLAB plugin - 2.6.1

- Cartool-3.91 / 6638 


\section{Detailed source localization steps}

The neural generators of each microstate were estimated. 6000 solution points distributed equally spaced in a grey matter-constrained head model of a child MRI brain volume template; the grey matter represented the solution space for which the electrical activity would be localized. The MRI template was an average of 82 4.5- to 8.5-year-old children from the National Institute of Health (NIH) funded MRI Study of Normal Brain Development (Fonov et al., 2011). The EEG sensor net template was co-registered to the MRI head model. The Local Spherical Model with Anatomical Constraints (LSMAC; Brunet et al., 2011) model calculated an adaptive local spherical model at each electrode. To do so, the thicknesses of the scalp, skull, CSF, and brain were estimated under each electrode. These thicknesses were then used in a 4shell spherical model with the local radiuses, allowing the real geometry between solution points and electrodes to be accounted for. The 4-Shell model represented the skull thickness and conductivity of a 5.5-year-old child, incorporating the scalp, skull, cerebrospinal fluid, and brain, and offering exact solutions for any number of isotropic spherical layers. A distributed linear inverse solution called LORETA (Low Resolution Brain Electromagnetic Tomography; PascualMarqui et al., 1994) was then calculated. The results were optimized with regularization, which considered the level of background EEG noise and enforced smoothness of the results, and were standardized to correct for the variability of EEG power observed at solution points across time (i.e., to eliminate activation biases; Michel \& Brunet, 2019). The norm or amplitude of dipoles was saved as scalar, positive values at each solution point and averaged across all timepoints for each microstate.

Each microstate's source map was thresholded to the solution points above the 95 th percentile of activations across all participants. Source maps were converted to volumes by 
computing all intermediate voxels from the grey matter-constrained head model using cubic splines. Importantly, no new maxima were artificially created. Volumes were imported to the NIH's open-source Analysis of Functional NeuroImages (AFNI; Cox, 1996) program and demeaned by subtracting the mean of all source maps from each source map to highlight microstate-specific sources (Custo et al., 2017). AFNI was also used to identify activated sources with the Talairach-Tournoux Atlas. 


\section{Detailed recruitment description}

After screening 323 children, 249 were eligible to participate in the overarching study. Of the 249 eligible children, 17 declined to participate, 13 were scheduled but cancelled due to COVID-19 concerns, and 48 did not complete the study for other reasons (e.g., did not show up

to their scheduled visit and never rescheduled). A total of 171 children completed their first study visit. However, resting state EEG was added to the study protocol approximately halfway through data collection. Participants who did not have EEG data at their first study visit but had EEG data at their second study visit were included. Two children were older than eight years of age and were excluded. Twenty-nine children completed their first study visit when EEG was not part of the study protocol, and they did not return for their second study visit. The number of eligible children who participated at a time when EEG was part of the study protocol was 140 . However, 32 children did not complete EEG because they refused to or could not wear the EEG sensor net or were noncompliant for other reasons. In sum, 108 participants completed an EEG session at either their first or second study visit. Of these, 103 provided adequate EEG data (see above). There were no significant differences between the age, number of males and females, and depressive symptom scores of the 140 eligible children who participated at a time when EEG was part of the study protocol and the 103 participants who provided adequate EEG data (see above). 
Since the primary aim of the overarching study was to investigate reward processing in early emerging risk for depression, we assessed Pearson's correlations between microstate parameters and children's depressive symptoms and found no statistically significant correlations (see below). Therefore, we did not control for children's depressive symptoms in statistical models.

Pearson's correlations between each microstate's temporal parameters and children's depressive symptoms.

\begin{tabular}{lcc}
\hline & Correlation $(r)$ & $p$ value \\
\hline Microstate 1 & & \\
GEV & .02 & .85 \\
Duration & .09 & .36 \\
Coverage & .06 & .55 \\
Occurrence & .05 & .60 \\
Microstate 2 & & \\
GEV & -.10 & .32 \\
Duration & -.10 & .31 \\
Coverage & -.10 & .34 \\
Occurrence & -.07 & .48 \\
Microstate 3 & & \\
GEV & -.004 & .97 \\
Duration & .005 & .96 \\
Coverage & .04 & .72 \\
Occurrence & .11 & .29 \\
Microstate 4 & & \\
GEV & -.005 & .96 \\
Duration & -.07 & .47 \\
Coverage & .001 & .99 \\
Occurrence & .01 & .90 \\
\hline
\end{tabular}

Note. Children's depressive symptoms are represented as the unstandardized residual values from a regression model in which the Beck Depression Inventory, 2nd edition (BDI-II; Beck et al., 1996) was entered as the independent variable and the Preschool Feelings Checklist, Scale Version (PFC-Scale; Luby et al., 2012) was entered as the dependent variable (these measures were collected at each study visit as part of the overarching study). This procedure reduced the inflation of children's depressive symptoms by maternal depressive symptoms. The sample size for this analysis was reduced to 100 because three participants did not have a PFC-Scale and/or BDI-2 score. 
For the Results section:

\section{Supplementary Figure 1}

Three-dimensional views of the four microstates.

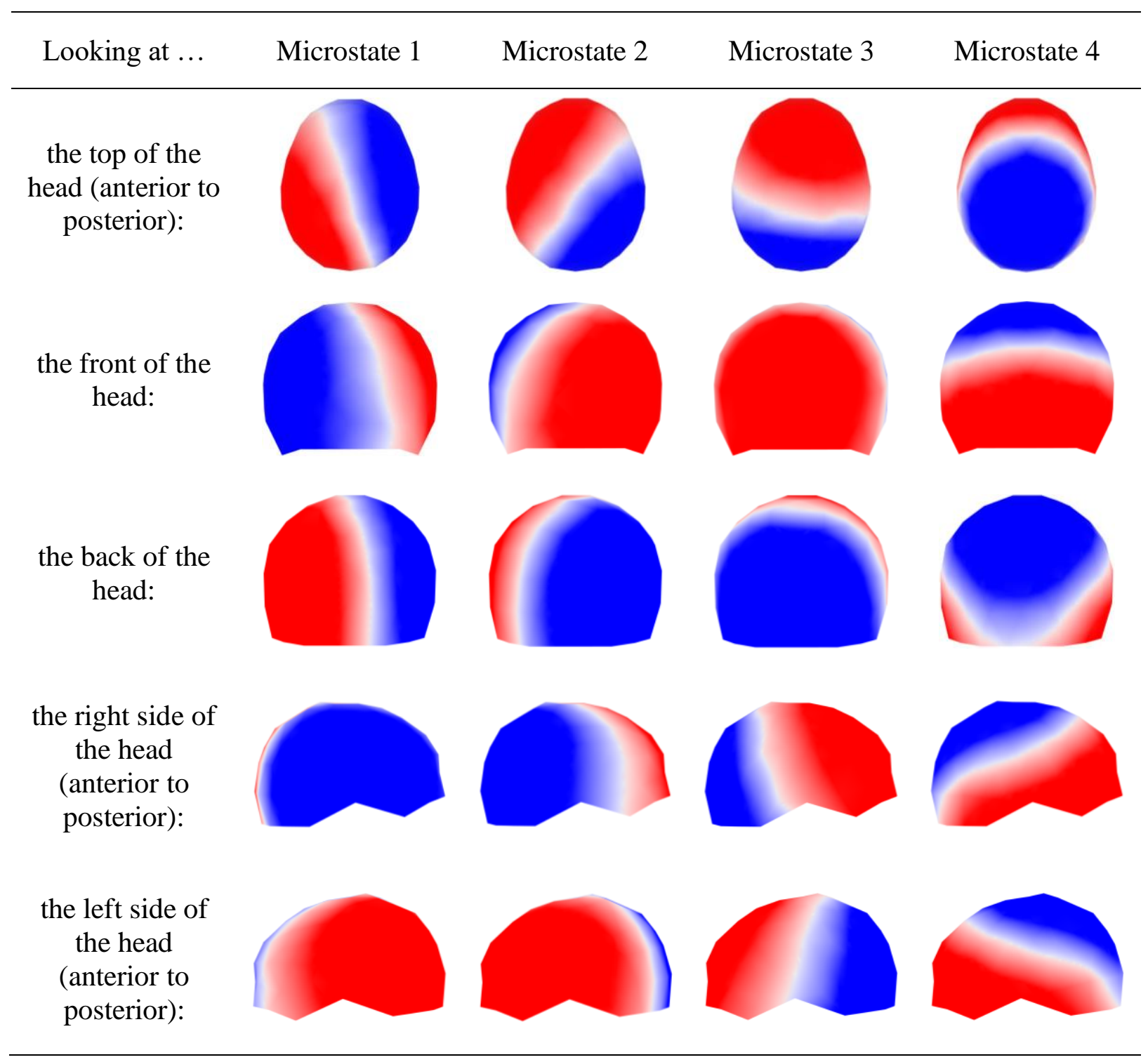

Note. Microstates were derived from a polarity-invariant clustering algorithm, 


\section{Supplementary Figure 2}

Spatial correlations between microstate topographies.

Microstate 1 Microstate 2 Microstate 3 Microstate 4

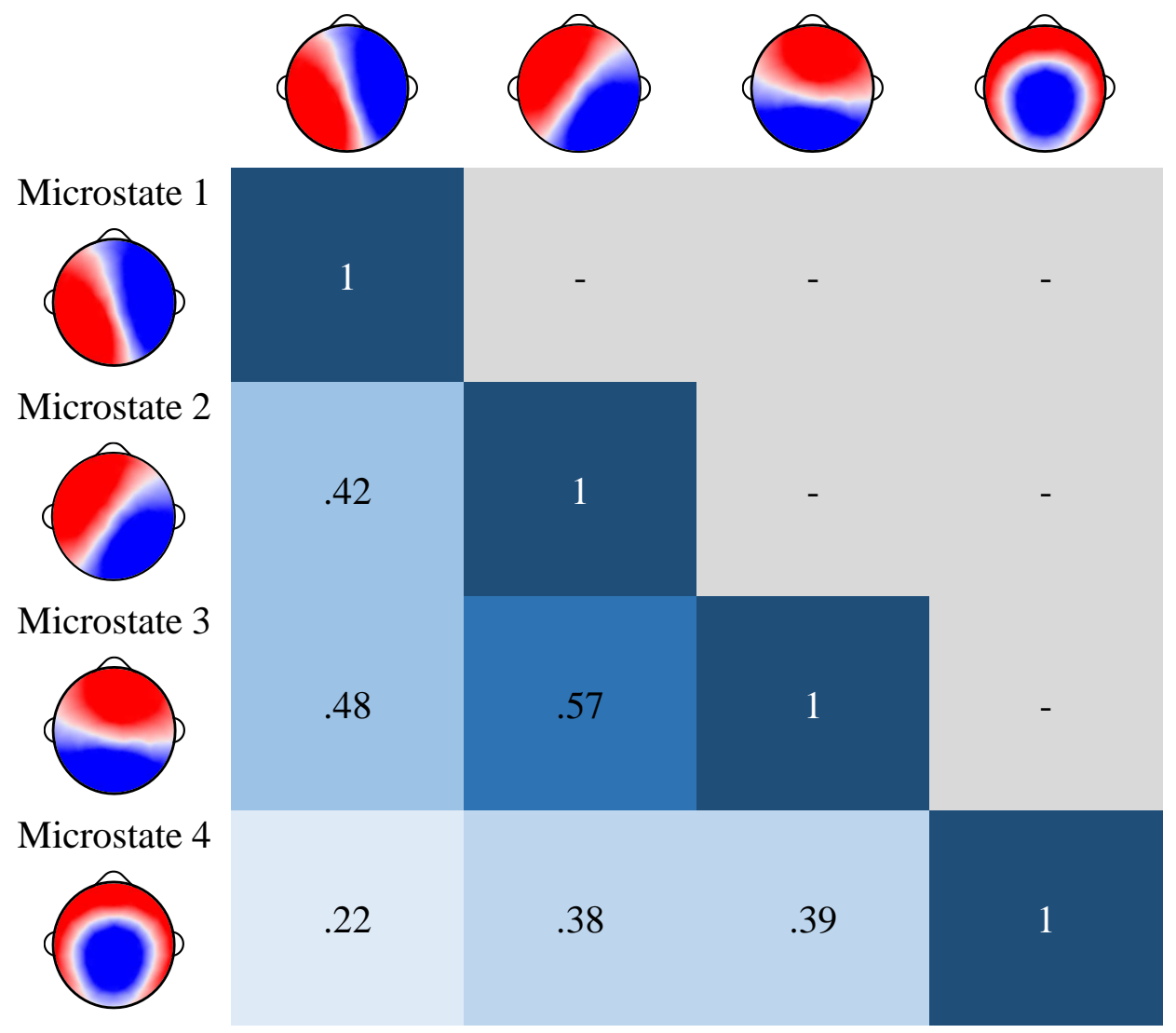

Note. Microstates were derived from a polarity-invariant clustering algorithm. 
Between-microstate differences in the means of their durations with outliers included

There was a significant difference in the mean duration between microstates, $F(1.98$, 201.79) $=206.66, p<.0001$, with a large effect size, $\eta^{2} g=0.64$. Planned post-hoc contrasts revealed that Microstate 1 duration was lower than Microstate 2 duration, $t(102)=-8.04, p<$ .0001 , and Microstate 3 duration, $\mathrm{t}(102)=-17.25, \mathrm{p}<.0001$, but higher than Microstate 4 duration, $\mathrm{t}(102)=6.54, \mathrm{p}<.0001$. Microstate 2 duration was lower than Microstate 3 duration, $\mathrm{t}(102)=-9.90, \mathrm{p}<.0001$, but higher than Microstate 4 duration, $\mathrm{t}(102)=12.43, \mathrm{p}<.0001$. Microstate 3 duration was higher than Microstate 4 duration, $\mathrm{t}(102)=19.87, \mathrm{p}<.0001$. The paired mean difference of microstate 2 minus microstate 1 duration was $6.71,95 \%$ CI [5.18, 8.37]; of microstate 3 minus microstate 1 duration was $21.70,95 \%$ CI [19.50, 24.00]; of microstate 4 minus microstate 1 duration was $-4.66,95 \%$ CI [-6.13, -3.23]; of microstate 3 minus microstate 2 duration was $15.00,95 \%$ CI [12.50, 17.50]; of microstate 4 minus microstate 2 duration was $-11.40,95 \%$ CI [-13.20, -9.70]; of microstate 4 minus microstate 3 duration was $26.40,95 \%$ CI $[-28.80,-24.00]$. 


\section{Supplementary Table 2}

Regressions examining age and sex effects of each microstate's temporal parameters including outliers.

\begin{tabular}{|c|c|c|c|c|c|c|c|c|c|c|c|c|c|c|c|c|}
\hline \multirow[b]{2}{*}{ Variable } & \multicolumn{4}{|c|}{ GEV } & \multicolumn{4}{|c|}{ Duration } & \multicolumn{4}{|c|}{ Coverage } & \multicolumn{4}{|c|}{ Occurrence } \\
\hline & $B$ & $S E B$ & $\beta$ & $p$ & $B$ & $S E B$ & $\beta$ & $p$ & $B$ & $S E B$ & $\beta$ & $p$ & $B$ & $S E B$ & $\beta$ & $p$ \\
\hline Microstate 1 & \multicolumn{4}{|c|}{$F(3,99)=2.13, \mathrm{R}^{2}=.03, p=.17$} & \multicolumn{4}{|c|}{$F(3,99)=0.88, R^{2}=-.003, p=.45$} & \multicolumn{4}{|c|}{$F(3,99)=1.92, R^{2}=.03, p=.17$} & \multicolumn{4}{|c|}{$F(3,99)=2.40, R^{2}=.04, p=.17$} \\
\hline (Intercept) & 0.09 & 0.003 & - & $<.001 * * *$ & 78.75 & 0.46 & - & $<.001 * * *$ & 19.66 & 0.41 & - & $<.001 * * *$ & 2.17 & 0.03 & - & $<.001 * * *$ \\
\hline Age & 0.003 & 0.003 & 0.10 & .30 & 0.41 & 0.43 & 0.09 & .35 & 0.31 & 0.38 & 0.08 & .42 & 0.01 & 0.03 & 0.03 & .75 \\
\hline Sex & 0.01 & 0.003 & 0.21 & $.03 *$ & 0.54 & 0.46 & 0.12 & .24 & 0.90 & 0.41 & 0.22 & $.03 *$ & 0.08 & 0.03 & 0.25 & $.01 *$ \\
\hline Age $*$ Sex & -0.002 & 0.003 & -0.08 & .40 & 0.18 & 0.43 & 0.04 & .67 & -0.20 & 0.38 & -0.05 & .60 & -0.02 & 0.03 & -0.06 & .52 \\
\hline (Intercept) & 0.16 & 0.01 & - & $<.001 * * *$ & 85.45 & 0.70 & - & $<.001 * * *$ & 26.97 & 0.59 & - & $<.001 * * *$ & 2.68 & 0.04 & - & $<.001 * * *$ \\
\hline Age & -0.004 & 0.005 & -0.08 & .44 & 0.21 & 0.66 & 0.03 & .76 & -0.22 & 0.56 & -0.04 & .69 & -0.03 & 0.03 & -0.09 & .37 \\
\hline Sex & 0.004 & 0.01 & 0.08 & .45 & 0.55 & 0.70 & 0.08 & .43 & 0.23 & 0.59 & 0.04 & .69 & -0.01 & 0.04 & -0.02 & .83 \\
\hline Age $*$ Sex & -0.01 & 0.005 & -0.13 & .20 & -0.41 & 0.66 & -0.06 & .54 & -0.72 & 0.56 & -0.13 & .20 & -0.04 & 0.03 & -0.13 & .20 \\
\hline Microstate 3 & \multicolumn{4}{|c|}{$F(3,99)=1.44, R^{2}=.01, p=.24$} & \multicolumn{4}{|c|}{$F(3,99)=2.80, R^{2}=.05, p=.09$} & \multicolumn{4}{|c|}{$F(3,99)=2.97, R^{2}=.05, p=.09$} & \multicolumn{4}{|c|}{$F(3,99)=2.11, R^{2}=.03, p=.14$} \\
\hline (Intercept) & 0.28 & 0.01 & - & $<.001 * * *$ & 100.85 & 1.06 & - & $<.001 * * *$ & 39.24 & 0.77 & - & $<.001 * * *$ & 3.17 & 0.03 & - & $<.001 * * *$ \\
\hline Sex & -0.01 & 0.01 & -0.17 & .09 & -2.62 & 1.06 & -0.24 & $.02 *$ & -2.18 & 0.77 & -0.27 & $.005 * *$ & -0.07 & 0.03 & -0.23 & $.02 *$ \\
\hline Age $*$ Sex & -0.003 & 0.01 & -0.04 & .71 & -0.09 & 1.00 & -0.01 & .93 & -0.39 & 0.72 & -0.05 & .59 & -0.01 & 0.03 & -0.05 & .64 \\
\hline Microstate 4 & \multicolumn{4}{|c|}{$F(3,99)=4.02, R^{2}=.08, p=.01^{*}$} & \multicolumn{4}{|c|}{$F(3,99)=3.70, R^{2}=.07, p=.01^{*}$} & \multicolumn{4}{|c|}{$F(3,99)=3.95, R^{2}=.08, p=.01^{*}$} & \multicolumn{4}{|c|}{$F(3,99)=3.84, R^{2}=.08, p=.01 *$} \\
\hline (Intercept) & 0.06 & 0.003 & - & $<.001 * * *$ & 74.08 & 0.57 & - & $<.001 * * *$ & 14.13 & 0.52 & - & $<.001 * * *$ & 1.67 & 0.05 & - & $<.001 * * *$ \\
\hline Age & -0.003 & 0.003 & -0.13 & .19 & -0.19 & 0.53 & -0.03 & .72 & -0.69 & 0.49 & -0.14 & .17 & -0.07 & 0.05 & -0.15 & .13 \\
\hline Sex & 0.01 & 0.003 & 0.24 & $.01 *$ & 0.67 & 0.57 & 0.11 & .24 & 1.04 & 0.52 & 0.19 & $.049 *$ & 0.11 & 0.05 & 0.20 & $.03 *$ \\
\hline Age $*$ Sex & 0.01 & 0.003 & 0.22 & $.03 *$ & 1.66 & 0.53 & 0.30 & $.002 * *$ & 1.31 & 0.49 & 0.26 & $.009 * *$ & 0.11 & 0.05 & 0.23 & $.02 *$ \\
\hline
\end{tabular}

Note. $R^{2}$ values were adjusted for three predictors. Full model $p$ values were Benjamini-Hochberg-corrected for four comparisons, but $p$ values at the predictor-level were not corrected: $* p<.05, * * p<.01, * * * p<.001$. 


\section{Supplementary Figure 3}

Mean of all four microstate source maps.

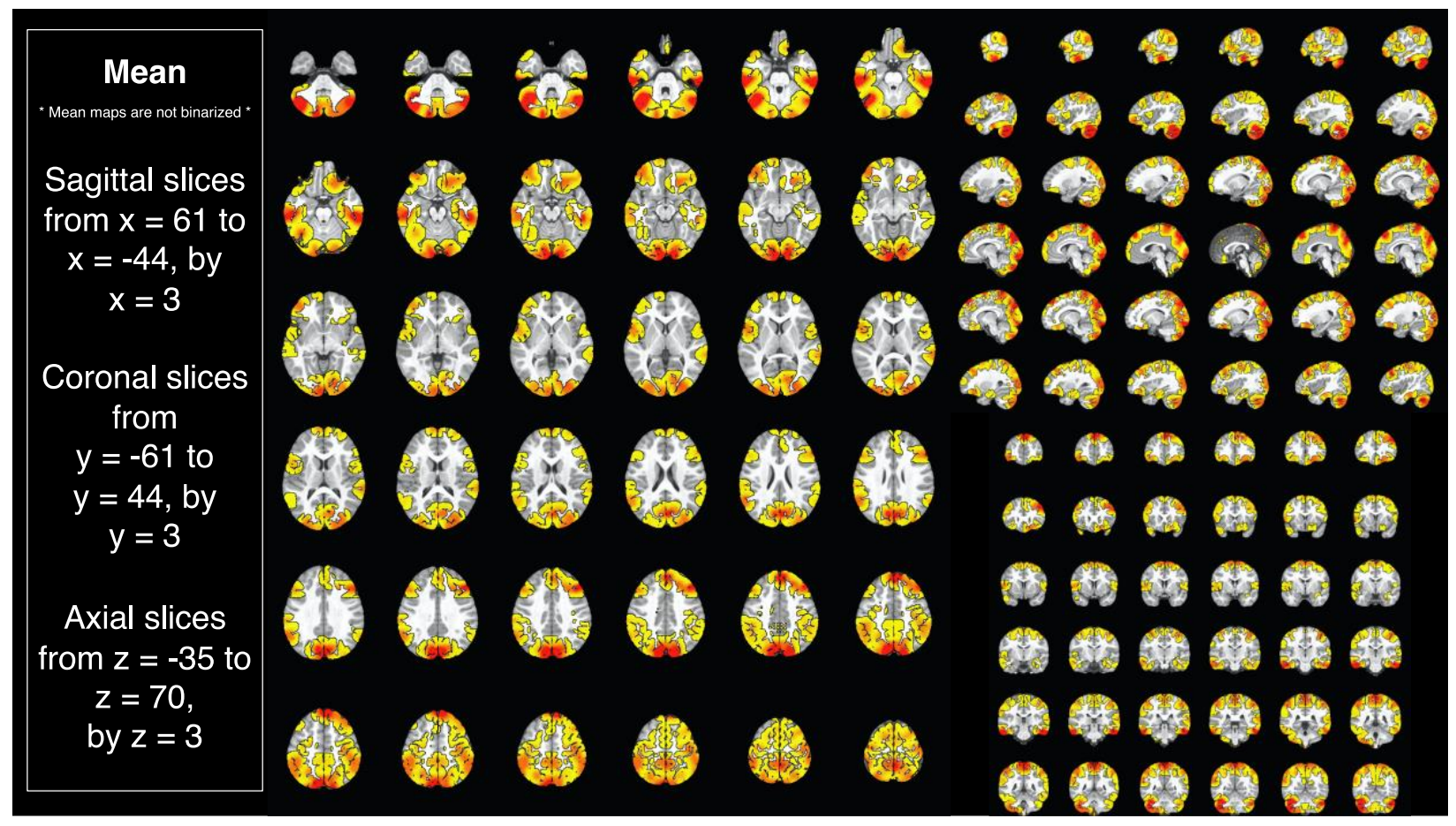




\section{Supplementary Figure 4}

Microstate 1 sources.

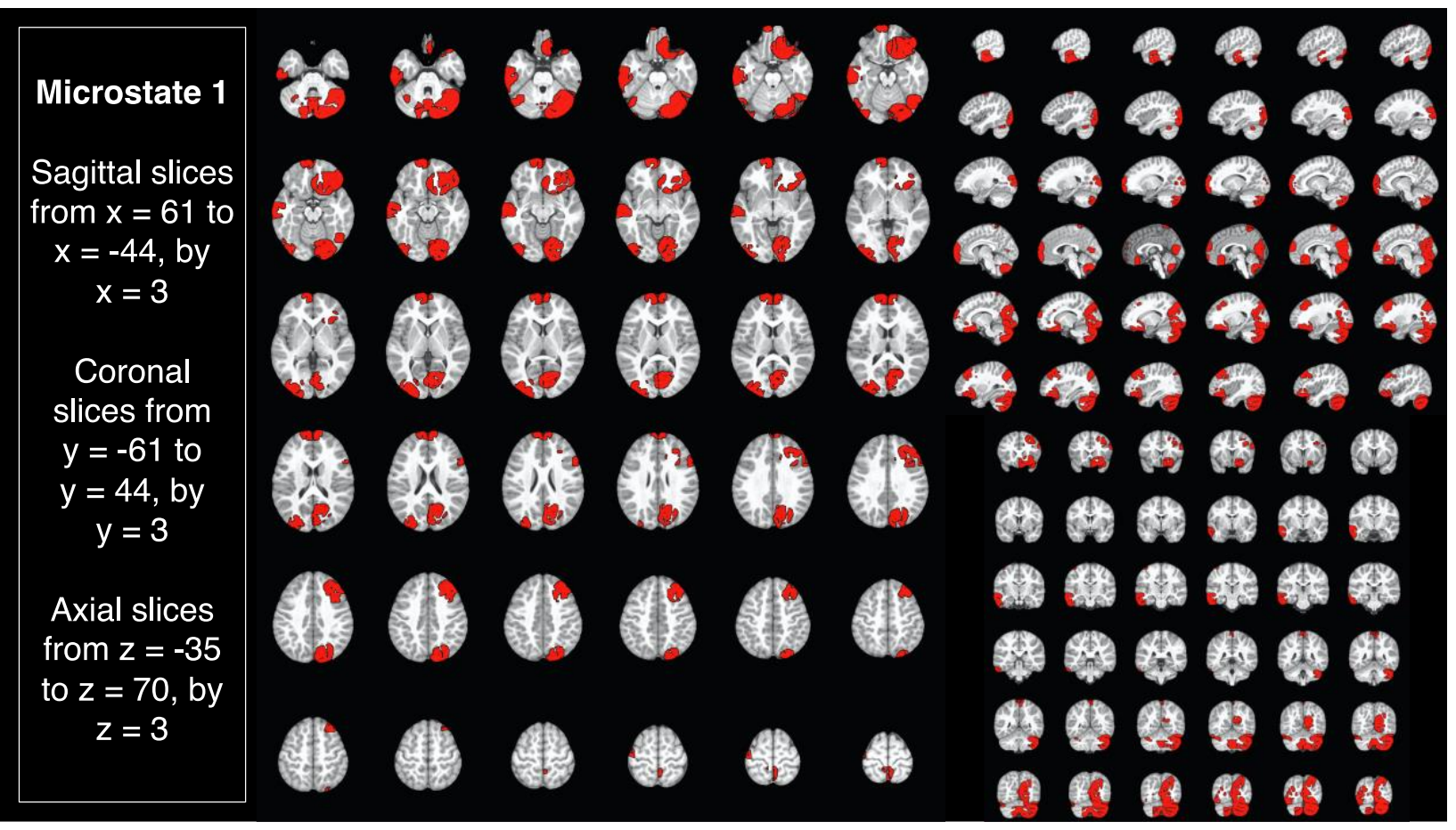




\section{Supplementary Figure 5}

Microstate 2 sources.

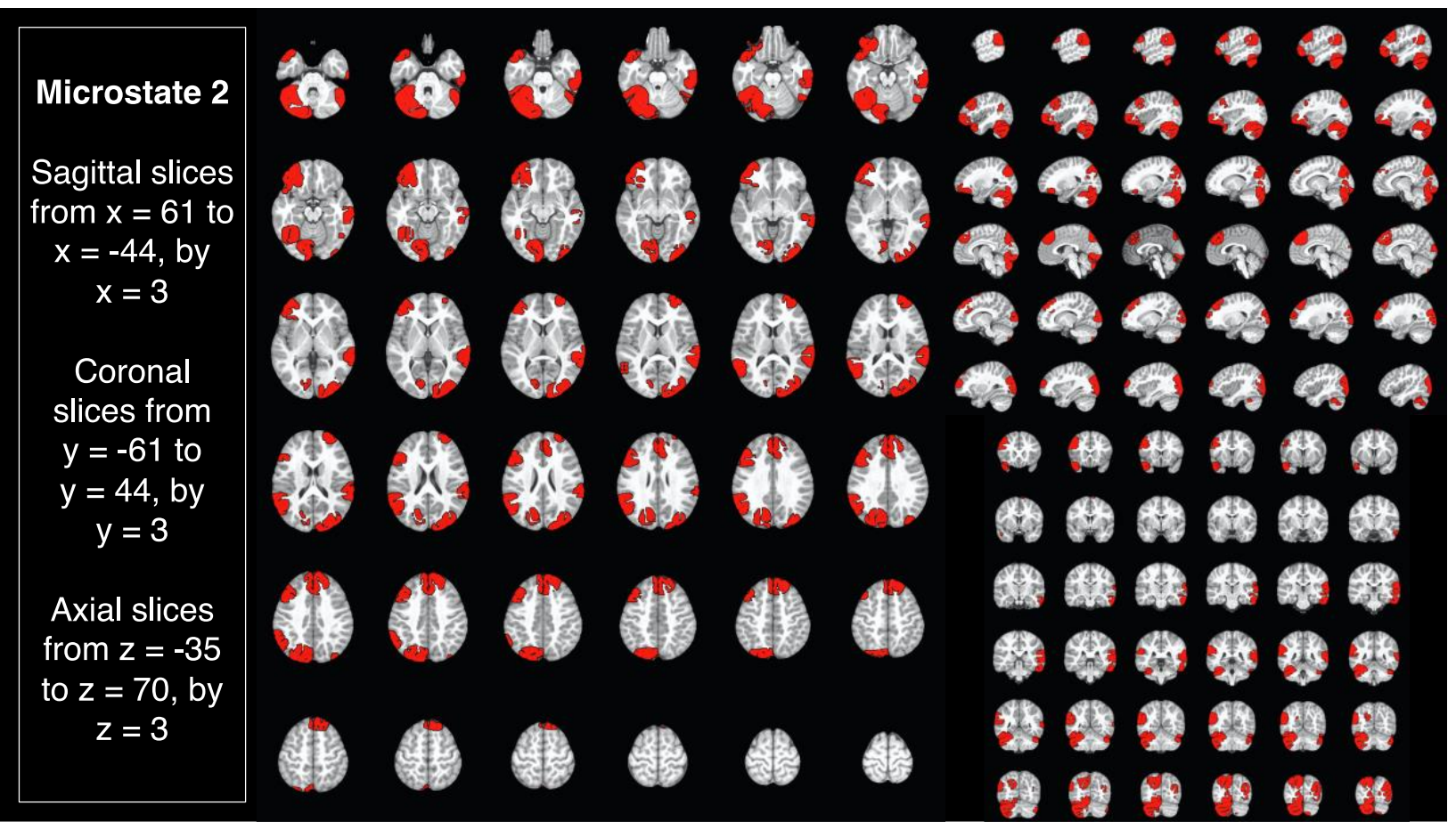


Supplementary Figure 6

Microstate 3 sources.

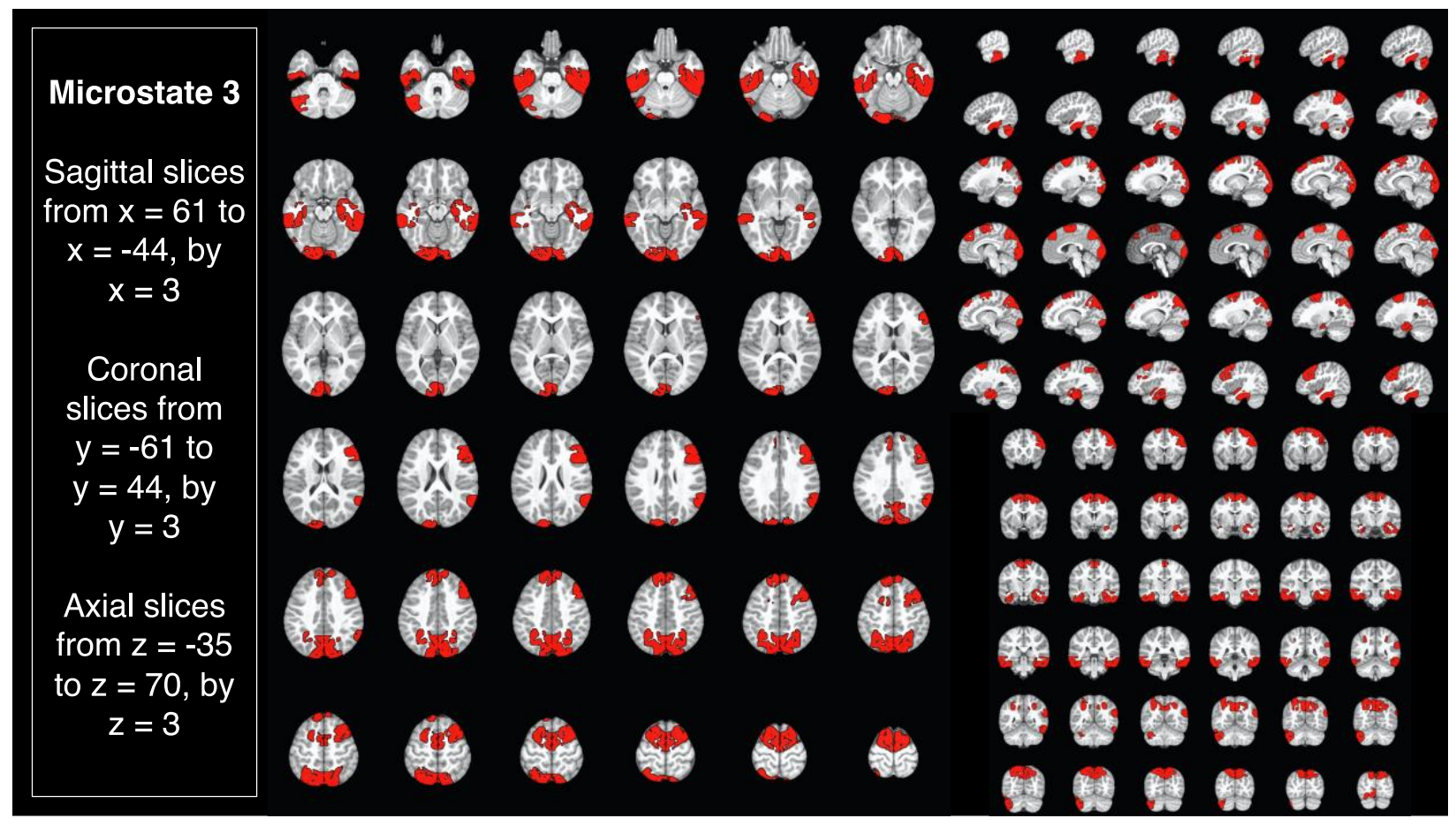




\section{Supplementary Figure 7}

Microstate 4 sources.

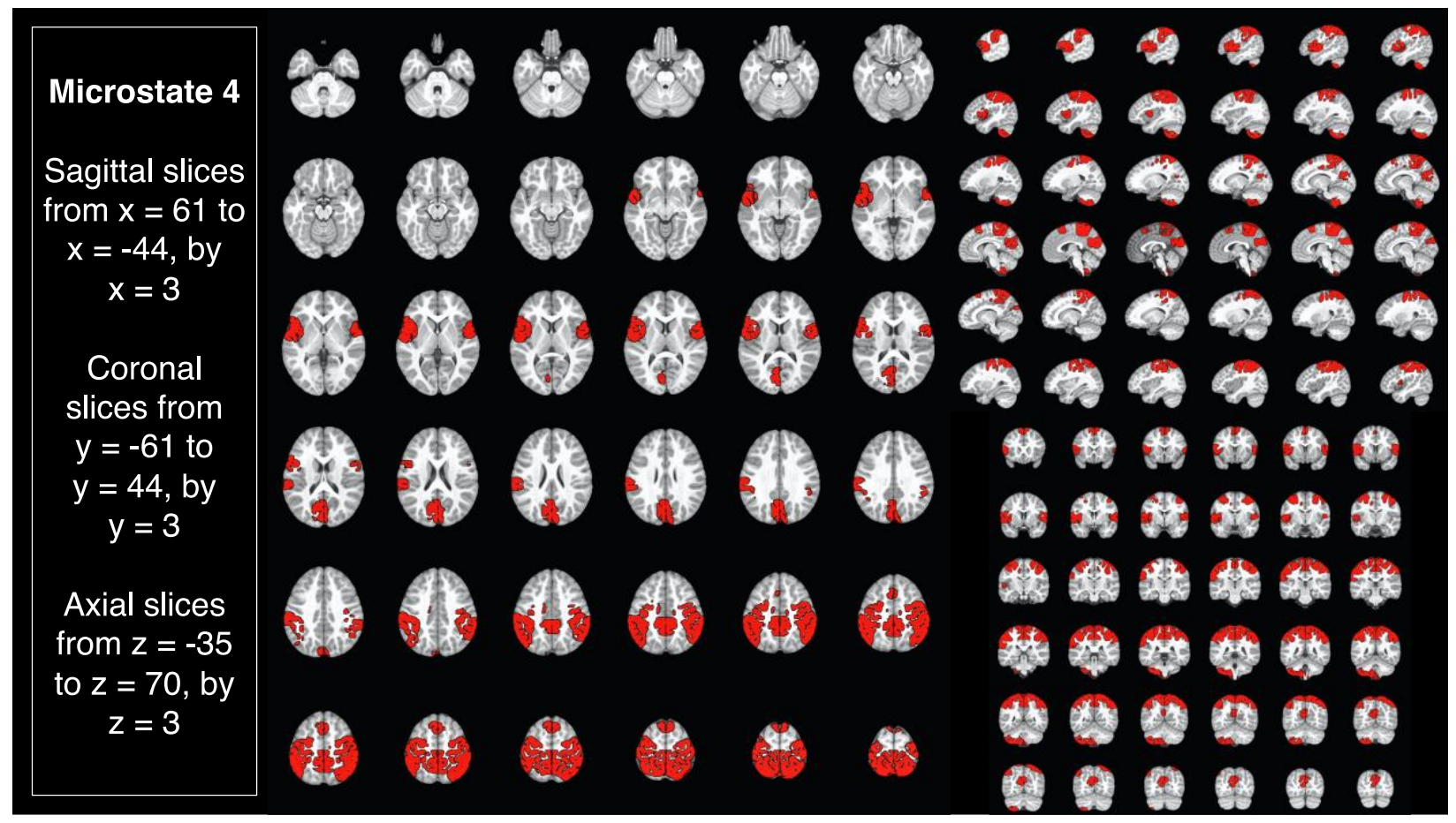




\section{References for the Supplementary Materials}

Beck, A. T., Steer, R. A., \& Brown, G. K. (1996). Beck depression inventory (BSI-II). Pearson, 10.

Ben-Shachar, M. S. (2018). TBT: Reject and interpolate channels on a epoch by epoch basis (2.6.1) [Computer software]. https://doi.org/10.5281/zenodo.1241518

Chang, C. Y., Hsu, S. H., Pion-Tonachini, L., \& Jung, T. P. (2018). Evaluation of Artifact Subspace Reconstruction for Automatic EEG Artifact Removal. Proceedings of the Annual International Conference of the IEEE Engineering in Medicine and Biology Society, EMBS, 2018-July, 1242-1245. https://doi.org/10.1109/EMBC.2018.8512547

Cox, R. W. (1996). AFNI: Software for Analysis and Visualization of Functional Magnetic Resonance Neuroimages. Computers and Biomedical Research, 29(3), 162-173. https://doi.org/10.1006/cbmr.1996.0014

Custo, A., Van De Ville, D., Wells, W. M., Tomescu, M. I., Brunet, D., \& Michel, C. M. (2017). Electroencephalographic Resting-State Networks: Source Localization of Microstates. Brain Connectivity, 7(10), 671-682. https://doi.org/10.1089/brain.2016.0476

Delorme, A., Sejnowski, T., \& Makeig, S. (2007). Enhanced detection of artifacts in EEG data using higher-order statistics and independent component analysis. NeuroImage, 34(4), 1443-1449. https://doi.org/10.1016/j.neuroimage.2006.11.004

Fonov, V., Evans, A. C., Botteron, K., Almli, C. R., McKinstry, R. C., \& Collins, D. L. (2011). Unbiased average age-appropriate atlases for pediatric studies. NeuroImage, 54(1), 313327. https://doi.org/10.1016/j.neuroimage.2010.07.033

Gabard-Durnam, L. J., Mendez Leal, A. S., Wilkinson, C. L., \& Levin, A. R. (2018). The Harvard Automated Processing Pipeline for Electroencephalography (HAPPE): 
Standardized Processing Software for Developmental and High-Artifact Data. Frontiers in Neuroscience, 12, 97. https://doi.org/10.3389/fnins.2018.00097

Lee, T.-W., Girolami, M., \& Sejnowski, T. J. (1999). Independent Component Analysis Using an Extended Infomax Algorithm for Mixed Subgaussian and Supergaussian Sources. Neural Computation, 11(2), 417-441. https://doi.org/10.1162/089976699300016719

Liu, J., Xu, J., Zou, G., He, Y., Zou, Q., \& Gao, J.-H. (2020). Reliability and Individual Specificity of EEG Microstate Characteristics. Brain Topography, 33(4), 438-449. https://doi.org/10.1007/s10548-020-00777-2

Luby, J., Lenze, S., \& Tillman, R. (2012). A novel early intervention for preschool depression: Findings from a pilot randomized controlled trial. Journal of Child Psychology and Psychiatry, 53(3), 313-322. https://doi.org/10.1111/j.1469-7610.2011.02483.x

Michel, C. M., \& Brunet, D. (2019). EEG Source Imaging: A Practical Review of the Analysis Steps. Frontiers in Neurology, 10, 325. https://doi.org/10.3389/fneur.2019.00325

Mullen, T. (2012). NITRC: CleanLine: Tool/Resource Info.

Mullen, T. R., Kothe, C. A. E., Chi, Y. M., Ojeda, A., Kerth, T., Makeig, S., Jung, T. P., \& Cauwenberghs, G. (2015). Real-time neuroimaging and cognitive monitoring using wearable dry EEG. IEEE Transactions on Biomedical Engineering, 62(11), 2553-2567. https://doi.org/10.1109/TBME.2015.2481482

Pascual-Marqui, R. D., Michel, C. M., \& Lehmann, D. (1994). Low resolution electromagnetic tomography: A new method for localizing electrical activity in the brain. International Journal of Psychophysiology, 18(1), 49-65. https://doi.org/10.1016/01678760(84)90014-X 
Piazza, C., Cantiani, C., Akalin-Acar, Z., Miyakoshi, M., Benasich, A. A., Reni, G., Bianchi, A. M., \& Makeig, S. (2016). ICA-derived cortical responses indexing rapid multi-feature auditory processing in six-month-old infants. NeuroImage, 133, 75-87. https://doi.org/10.1016/j.neuroimage.2016.02.060

Pion-Tonachini, L., Kreutz-Delgado, K., \& Makeig, S. (2019). ICLabel: An automated electroencephalographic independent component classifier, dataset, and website. NeuroImage, 198, 181-197. https://doi.org/10.1016/j.neuroimage.2019.05.026 